\title{
Estimating Experienced Racial Segregation in U.S. Cities Using Large-Scale GPS Data
}

\author{
Susan Athey, Stanford University and NBER* \\ Billy Ferguson, Stanford University \\ Matthew Gentzkow, Stanford University and NBER \\ Tobias Schmidt
}

November 2021

\begin{abstract}
We estimate a measure of segregation, experienced isolation, that captures individuals' exposure to diverse others in the places they visit over the course of their days. Using Global Positioning System (GPS) data collected from smartphones, we measure experienced isolation by race. We find that the isolation individuals experience is substantially lower than standard residential isolation measures would suggest, but that experienced and residential isolation are highly correlated across cities. Experienced isolation is lower relative to residential isolation in denser, wealthier, more educated cities with high levels of public transit use, and is also negatively correlated with income mobility.
\end{abstract}

Classification: Social Sciences, Economic Sciences.

Keywords: segregation, race, GPS, smartphone data, isolation index, urban economics, inequality.

*E-mail: athey@stanford.edu, billyf@stanford.edu, gentzkow@stanford.edu, tobyschmidt@web.de. We thank Jonathan Dingel, Jessie Handbury, and numerous seminar participants for helpful inputs and suggestions. We also thank our many dedicated research assistants for their contributions to this project. We acknowledge funding from the Stanford Institute for Economic Policy Research (SIEPR). 


\section{Introduction}

Social outcomes are profoundly shaped by the extent to which groups are segregated from one another (Cutler and Glaeser 1997; Chetty and Hendren 2018a, 2018b; Chetty et al. 2016). As a result, large literatures have developed in economics, sociology, and related fields seeking to measure the extent of segregation across space and time.

Most of this empirical work focuses on segregation in where people live. A leading measure is the isolation index, which captures the share of individuals' neighbors who come from their own group 11 If we view the object of interest as the exposure of one group to another (Massey and Denton 1988; Cutler et al. 1999; Echenique and Fryer 2007), residential measures have obvious limitations. Individuals living in highly segregated neighborhoods may be exposed to diverse others where they work, shop, and socialize, while those living in apparently mixed neighborhoods may have little contact with their neighbors and commute to highly segregated places. A corollary is that standard residential segregation measures are highly sensitive to the way in which neighborhood boundaries are defined (Cowgill and Cowgill 1951; Massey and Denton 1988).

An important exception to this residential focus is a body of work in sociology characterizing the activity space of individuals -- the set of places individuals encounter as they move through their everyday lives 2 Researchers leverage surveys (Wong and Shaw 2011, Jones and Pebley 2014, Browning et al. 2017) and geolocation data (Shelton et al. 2015, Zhang et al. 2019, Phillips et al. 2021, Lee et al. 2021) to characterize the activity space for particular groups of people and the degree to which activity spaces overlap across groups.

In this paper, we build on this work to estimate an activity-based measure of segregation for a large sample of US cities using Global Positioning System (GPS) data. This experienced isolation has the same form as the isolation index, but rather than assuming individuals are exposed uniformly to those in their neighborhood of residence, it averages exposure over the places individuals actually visit over the course of their days. This measure does not depend on arbitrary neighborhood boundaries, and it takes explicit account of the diversity experienced away from home. It can capture individual-level heterogeneity within neighborhoods (Echenique and Fryer 2007), and it can be disaggregated across times of day, locations, and activities, thus giving a richer picture of the forces that increase or decrease segregation.

Our main data are GPS signals from a sample of US smartphone users covering approximately 5\% of the US population in the first four months of 2017. The data are obtained from a company that aggregates anonymous pings from a range of smartphone apps. We observe each device's home location as well as the location of every ping by the device recorded in the data. We map these locations to a grid of geographic units approximately 500 feet square, known as geohash7s. The sample of individuals is not random but is reasonably close to representative along a number of dimensions, and has sufficient coverage that we can correct for deviations from representativeness using sample weights. We use the movement patterns we observe to compute experienced racial isolation.

Because we do not observe an individual's race directly, we define the two types whose segregation we study to be individuals with homes in majority white geohash7s and individuals with homes in majority non-white geohash7s. We refer to these two groups as WDs (White home geohash7 Devices) and NWDs (Non-White home geohash7 Devices) for simplicity. The median share white of majority white and non-white home geohash7s are 0.89 and 0.22 , respectively. We discuss below the implications of using these geographic definitions in place of individual race, and we show robustness to an alternative strategy that imputes race at the individual level.

We present four main results: First, peoples' experiences as captured by our measure are substantially less isolated from diverse others than traditional residential isolation would suggest. The average experienced isolation across all Metropolitan Statistical Areas (MSAs) is 0.46, compared to average residential isolation of 0.61 3 This

\footnotetext{
${ }^{1}$ See, for example, Cutler and Glaeser (1997), Cutler et al. (1999), Gentzkow and Shapiro (2011), and Davis et al. (2019).

${ }^{2}$ See Cagney et. al (2020) for a recent literature review of the activity space literature.

${ }^{3}$ Residential isolation based on our geographic definition of WD and NWD is larger than the standard measure of residential isolation based
} 
implies that the share of WD's exposures to other WDs is 46 percentage points greater than the share of NWD's exposures to WDs.

Second, experienced and residential isolation across MSAs are highly correlated. The overall correlation of the two measures among the $366 \mathrm{MSAs}$ in our sample is 0.86. Among the 50 most populous MSAs, Milwaukee, WI; Detroit, MI; and Cleveland, OH rank in the top 5 in both residential and experienced isolation. Portland, OR; Seattle, WA; and Raleigh, NC rank in the bottom 5 for both measures.

Third, the variation in experienced relative to residential isolation is systematic. Experienced isolation is relatively lower in MSAs with higher population density and public transit use, consistent with the view that urban areas facilitate diverse interactions (Jacobs 1961). Experiences are also less isolated in MSAs with higher income and education and lower unemployment, possibly reflecting a role for social capital in reducing segregation (Putnam 2000). Finally, relative experienced isolation is negatively correlated with Chetty et al.'s (2014) measure of income mobility, consistent with both diverse interactions increasing mobility and with areas that facilitate opportunity also promoting diverse interactions.

Fourth, decompositions across time and space reveal the extent to which different activities increase or decrease segregation. Experienced isolation is lowest during the day and highest in the morning and evening. Experienced isolation in home neighborhoods is higher than residential measures would suggest, whereas experienced isolation outside of home neighborhoods is much lower. Isolation is lowest at entertainment, retail, and eating establishments, while time at locations like churches and schools is somewhat more isolated.

These findings have several broader implications. They suggest that standard measures understate the frequency of exposure to diverse others in the United States, and they highlight important forces such as commercial activity that increase it. They suggest that residential measures may nevertheless be a good proxy when the main goal is to assess relative levels of segregation across cities. Finally, they suggest nuances to keep in mind when assessing where the negative effects of segregation are likely to be largest. For example, local public goods such as schools or police services that are explicitly tied to residential boundaries may be more likely to be provided in segregated environments ${ }^{4}$ Children, those who do not work, and others whose daily life is more tied to their local neighborhoods are even less likely to be exposed to diverse others than current measures would suggest. Policies which affect the spatial distribution of commercial or leisure activities, or the transportation cost of accessing these activities, may be important tools for changing the distribution of exposure.

An important limitation to keep in mind in assessing all such implications is that we can only observe when devices occupy the same geographic space, not actual interaction between individuals. Under our construction, a restaurant-goer is just as exposed to the waiter or the cook in the kitchen as she is to the person sitting across the table. White (1983) highlights this subtlety by distinguishing geographic segregation (the concept we measure) and sociological segregation (based on actual interactions). While Sunstein (2002) and others argue that geographic segregation in this sense is of interest on its own 5 there are many reasons to think that the kind of exposure with limited interaction that occurs in places like shops and restaurants may have less social benefit than more sustained interpersonal contact.

We also emphasize two other important limitations of our analysis. First, we have no direct information about the individuals whose devices we see in our data, and so we define individual types based on the demographic

\footnotetext{
on individual race. We discuss the reasons for this difference below.

${ }^{4}$ Regarding policing and crime, Sampson and Levy (2020) find that despite activity-based measures of segregation differing from residential measures, activity-based measures do not seem to mediate the influence of residential segregation on the degree of violence experienced by communities.

${ }^{5}$ Sunstein (2002) writes that integrated physical spaces increase "the set of chance encounters with diverse others" and foster environments where "exposure is shared." He argues that overhearing conversations while at a restaurant, a bus stop, or just walking down the street all contribute to individuals' understanding of diverse others and open up opportunities for interaction.
} 
composition of home geohash $7 \mathrm{~s}$ rather than based on individual race. This means we are targeting a slightly different concept than much of the prior literature on segregation. We discuss alternative approaches including imputing race at the individual level in the Online Appendix. Second, our sample is not fully representative, and the geolocation information we get about any given device is sparse.

This paper builds on a large literature on measuring urban segregation. Important early work on both the definition and measurement of segregation includes Duncan and Duncan (1955), Taeuber and Taeuber (1965), White (1983), Massey and Denton (1988), and Massey and Denton (1993). Cutler et al. (1999) provide a comprehensive analysis of segregation in US cities over the century from 1890 to 1990. Card et al. (2008) study the dynamics of neighborhood tipping and Allcott et al. (2019) examine high and low-income neighborhood proximity to supermarkets and health inequality.

Our work is most related to the growing activity space literature on racial segregation, particularly Wong and Shaw (2011), Phillips et al. (2021), and Sampson and Levy (2020). Our measure is closely related to Wong and Shaw's (2011) extension of the exposure-based isolation index to activity beyond residential interaction. Phillips et al. (2021) use geotagged Twitter data to investigate racial segregation in 50 major cities based on mobility flows between neighborhoods. Sampson and Levy (2020) use the Phillips et al. (2021) estimates to similarly find that residential segregation is highly correlated with activity-based segregation. We extend prior work in that literature in several respects.

First, we estimate activity-based racial segregation across a larger set of U.S. cities (366) and a larger sample of individuals ( $>17$ million) than prior work. Second, we statistically estimate the correlation of city characteristics with the divergence between residential and activity-based measures of racial isolation. Third, we take seriously the concern Wong and Shaw (2011) voice about how to incorporate length of activity in measuring exposure by introducing a set of weights and exploring the robustness of such choices. Fourth, we provide decompositions of racial segregation by hour of day and by geographic features of a city such as parks, churches and restaurants ${ }^{6}$

Our work is also related to a broader literature using GPS or similar location data to study social interactions $7^{7}$ In particular, Moore and Reeves (2020) use a small geolocation dataset to motivate the need for dynamic measurement of racial and ethnic segregation 8

\section{Data}

\subsection{Geography}

We follow the literature in characterizing segregation at the level of MSAs and in using census tracts to approximate neighborhoods within MSAs 9 The finest geographic unit in our analysis is the geohash7, which as mentioned above is a unit of a grid roughly 500 feet square ${ }^{10}$ We use census blocks to impute geohash7 demographics. Appendix Figures S1, S2, and S3 illustrate the relative sizes of geohash7s, census blocks, and census tracts, focusing on an urban census tract and a rural census tract respectively in Birmingham, AL.

\footnotetext{
${ }^{6}$ Cagney et al. (2020) in their review of activity space approaches identify explorations of such decompositions as an "enduring question" for the literature.

${ }^{7}$ Glaeser et al. (2018) anticipate the value of such data. Blattman et al. (2018) track police patrols in Bogotà, Colombia using GPS to estimate how increased state presence affects violent and property crime. Chen and Rohla (2018) and Chen et al. (2019) use GPS data to measure the effects of political polarization on the length of Thanksgiving dinners and to measure racial differences in waiting times at polling places respectively. Davis et al. (2019) use data from Yelp to measure the segregation of restaurants in New York City, finding that restaurants are less segregated than residential neighborhoods. Caetano and Maheshri (2019) use data provided by the app Foursquare to quantify segregation by gender and by age in public places, and Phillips et al. (2019) use geotagged tweets to build an index capturing the extent to which residents in each neighborhood of a city travel to all other neighborhoods in equal proportions.

${ }^{8}$ Park and Kwan (2018) define a notion of "multi-contextual segregation" that is closely related to our work in considering segregation over the varying geographic and temporal contexts of peoples' daily lives.

${ }^{9}$ We omit Micropolitan Statistical Areas.

${ }^{10}$ The geohash geocoding scheme divides the globe into grids of increasing fineness. Geohash1s divide the globe into 32 cells of equal size. Geohash2s divide each of these cells into 32 smaller cells, and so on.
} 
We obtain information about the location of establishments and features of interest from two sources: InfoUSA and OpenStreetMaps (OSM). The 2015 InfoUSA US Businesses mailing list contains the names, addresses, industries, and latitude / longitude for 15.6 million businesses in the United States. We take from the full list all establishments that belong to the broad categories of "restaurants and bars," "civil, social and religious organizations," "accommodation," "sports and recreation," "entertainment," and "retail,' 11 2,368,216 places all in all. We match each establishment to the geohash7s that contain its latitude / longitude. From OSM, we extract polygon data for outdoor spaces like parks, playgrounds, sports fields and gardens, and educational institutions like schools, kindergartens, universities and colleges (See Appendix Section 2.1 for details). We associate each OSM feature with all geohash7s that intersect the feature's polygon. Appendix Figure $\mathrm{S} 4$ depicts geohash7s associated with civil, social, and religious organizations, education, outdoor spaces and restaurants and bars in downtown Birmingham, AL.

Many geohash7s are labelled with multiple features. We assume pings in a device's home geohash7 (defined below) are at home regardless of what other features are present. We assign all pings in non-home geohash7s that contain transportation features to transportation 12 All other pings are allocated uniformly across features present in the geohash7.

\subsection{GPS Device Movements}

Our GPS data are provided by a company that collects anonymous location data from mobile applications on users' smartphones. The sample is an unbalanced panel of GPS "pings" from more than 17 million devices spanning January to April 2017 13 Pings are logged whenever an application on a device requests location information. In some cases this will be the result of a device actively using an application, such as for navigation or weather information, while in other cases applications may request the information even while running in the background. Pings thus occur at irregular intervals. For each ping, we observe a timestamp, a device identifier, and the geohash7 in which the ping occurs. The data also contain the geohash7 of each device's home, inferred probabilistically from the device's nighttime and early-morning pings.

\subsection{Demographics}

We impute geohash7 demographics from the 2010 census. We match each home geohash7 to the census tract that contains its centroid. This yields a matching tract for 99.53 percent of devices in our sample. We match each home geohash7 to all census blocks that overlap its area. This yields a match to at least one census block with non-zero population for 98.12 percent of devices. We assign demographics to each home geohash7 by taking an area-weighted average of the demographics of the overlapping blocks ${ }^{14}$ We define "white" population based on the census designation "White Alone (Non-Hispanic)," and we group all other census race groups in the category "non-white."

We use data on MSA characteristics from the 2010 American Community Survey (ACS) and the 2010 decennial census. These variables include the MSA's median age, education level, unemployment rate, median income, population density, and share of residents using public transit to get to work 15 We also use economic mobility measures from Chetty et al. (2020) indicating the share of individuals born to parents at the 25 th percentile of the income distribution who make it to the top quintile for white and black populations. We compute MSA-level mobility measures by averaging across counties weighting by white and black county populations respectively.

\footnotetext{
${ }^{11}$ See Appendix Section $[\mathrm{S} 1$ for our manual classification of NAICS code into these categories.

${ }^{12}$ In Appendix Section S2.1 we show that our results are robust to both dropping pings in transportation features and dropping sequential pings that are travelling over $12 \mathrm{mph}$.

${ }^{13}$ We use "GPS" as a shorthand for a variety of means used by smartphones to determine their physical location. These include cell phone towers, the identity of nearby WiFi networks as well as the US GPS and the Russian GLONASS systems of satellites.

${ }^{14}$ We show robustness to alternative methods of demographic imputation in Appendix $\mathbf{S} 2.2$

${ }^{15}$ See Appendix Table S4 for a complete description and sources for census, ACS, and mobility variables.
} 


\subsection{Summary Statistics}

We observe 17,730,615 devices with home locations identified in 7,292,623 distinct geohash7s. We match these home geohash7s to 72,785 census tracts and 6,186,564 census blocks. This matching procedure succeeds for $17,397,580$ devices, the final sample used throughout the rest of the paper.

To assess the representativeness of the sample, we compare the average census tract demographics of devices in our sample to averages in the US population. We find that our sample is representative in terms of gender, age, and unemployment rate. We find that it slightly oversamples more educated and wealthy areas, with average median income across census tracts in our sample about a thousand dollars more than the U.S. mean, and census tract poverty rate about a percentage point lower. We address this imbalance by weighting as shown in equation $44^{16}$ Details of this comparison and summaries of the average activity levels of devices in our sample are shown in Appendix Tables S2 2 and S3 respectively.

While our WD and NWD designations are not equivalent to individual race, they are highly correlated with it. The median share white in a device's home geohash7 is 0.22 for NWDs and 0.89 for WDs. We plot the histogram of this share for both groups in Appendix Figure $\mathrm{S} 8$

\section{Measure}

\subsection{Definition}

Consider a population of individuals indexed by $i$ and a set of MSAs or other geographic areas of interest indexed by $a$. We collect each individual who is a member of one of two groups which we denote $W$ and $N W$. In our analysis below, $W$ will be individuals from majority white geohash7s (WDs) and $N W$ will be individuals from majority non-white geohash7s (NWDs). Each individual has a set of exposures to other individuals in area $a$. We let $e_{i} \in[0,1]$ denote the share of individual $i$ 's exposures that are to members of group $W{ }^{17}$

A general form of the isolation index for area $a$ captures the difference between the average value of $e_{i}$ among individuals in the two groups (cf. Gentzkow and Shapiro 2011):

$$
I_{a}=\frac{1}{\left|W_{a}\right|} \sum_{i \in W_{a}} e_{i}-\frac{1}{\left|N W_{a}\right|} \sum_{i \in N W_{a}} e_{i}
$$

Here $W_{a}$ and $N W_{a}$ are the sets of individuals making up the two groups in area $a$ and $|\cdot|$ denotes the size of these sets. This measure ranges from zero-no isolation, with average $e_{i}$ equal for the two groups-to one-perfect isolation, with $e_{i}=0$ for all $i \in N W$ and $e_{i}=1$ for all $i \in W$.

The standard version of this measure is residential isolation, which is equivalent to equation (1) under the assumption that each individual is exposed uniformly to others in her neighborhood of residence (Massey and Denton 1988; Cowgill and Cowgill 1951; Jahn 1950). In practice neighborhoods are typically defined to be census tracts. Letting $c(i)$ denote $i$ 's census tract of residence, and letting $r_{c}$ denote the share of the residents of tract $c$ who are in

\footnotetext{
${ }^{16}$ In Appendix Figure S7 we compare the MSA device weighted (using weights 4 share white to the true share white of each MSA and find that the device weights effectively recover the true demographic shares.

${ }^{17}$ In our empirical analysis, we focus on the case where the groups $W$ and $N W$ partition the population, so that $1-e_{i}$ is individual $i$ 's exposure to members of group $N W$. Our measure is also well-defined in the case where some individuals in the population are neither in $W$ nor $N W$. In this case, isolation where $e_{i}$ is the share exposed to $W$ may be different from isolation had we defined $e_{i}$ as the share exposed to $N W$.
} 
group $W$, residential isolation is given by: 18

$$
R I_{a}=\frac{1}{\left|W_{a}\right|} \sum_{i \in W_{a}} r_{c(i)}-\frac{1}{\left|N W_{a}\right|} \sum_{i \in N W_{a}} r_{c(i)} .
$$

Because this measure does not rely on any information other than the racial composition of each neighborhood, it can easily be computed using aggregate census data.

The measure we define, experienced isolation, instead assumes that $e_{i}$ is given by the composition of the individuals actually present in the locations that $i$ visits over time. We index time by $t \in[0,1]$ and consider a finite set of locations within area $a$ indexed by $l$. We think of a location $l$ as a specific place such as a restaurant, workplace, or park that is much smaller than a neighborhood. In our application, locations will be geohash7s. Letting $l(i, t)$ denote $i$ 's location at time $t$, and letting $s(l, t)$ denote the share of individuals in location $l$ at time $t$ who are from group $W$, experienced isolation is defined to be: ${ }^{19}$

$$
E I_{a}=\frac{1}{\left|W_{a}\right|} \sum_{i \in W_{a}} \int_{t=0}^{1} s(l(i, t), t) d t-\frac{1}{\left|N W_{a}\right|} \sum_{i \in N W_{a}} \int_{t=0}^{1} s(l(i, t), t) d t .
$$

\subsection{Estimation}

Estimating experienced isolation $E I_{a}$ would be straightforward if we observed continuous location data for all individuals. While our GPS dataset is rich, it still falls well short of this ideal. There are two key limitations: (1) we observe locations only when a device pings rather than continuously; (2) we only observe a sample of individuals not the full population. We make several simplifying assumptions in order to address these limitations.

To address (1), we first assume that the times when an individual $i$ visits a location $l$ are not systematically selected to be times when $s(l, t)$ is unusually high or low. That is, letting $\bar{s}_{l}$ denote the overall expectation of $s(l, t)$ over $t \in[0,1]$, we have $E[s(l, t) \mid l(i, t)=l]=\bar{s}_{l}$ for all $i$. Provided this assumption holds, the expectation of the term $\int_{t=0}^{1} s(l(i, t), t) d t$ is equal to $\bar{S}_{i}=\sum_{l} q_{i l} \bar{s}_{l}$ where $q_{i l}$ is the expected share of $i$ 's time that is spent in location $l$. We further assume that the times at which we observe pings are a random sample from $[0,1]$ so we can estimate $q_{i l}$ and $\bar{s}_{l}$ by the shares of $i$ 's pings that occur in location $l$ and the share of all pings in location $l$ that come from $W$ 's respectively.

Both of these are strong assumptions. The first would be violated, for example, if type $W$ individuals tend to visit a particular park or restaurant in the morning while type $N W$ individuals tend to visit it in the evening. Appendix Section $\mathrm{S} 2.4$ relaxes this assumption by defining locations $l$ at the location-hour level and yields very similar results to our baseline specification. The second would be violated if our data oversample periods in which the relative share of type $W$ individuals is unusually high or low. In Appendix Section $\mathrm{S} 2.3$ we present robustness to an alternative specification allowing non-random weighting of pings across time.

To address (2), we re-weight home locations in our sample to match the distribution of population in the 2010 census. Because our data are relatively sparse at the geohash7 level, we re-weight by census tract. We define the

\footnotetext{
${ }^{18}$ This form of the isolation index is equivalent to Gentzkow and Shapiro (2011). Much of the literature using the isolation index studies simply the exposures of a group, without taking their difference. (White 1986, Iceland et al. 2002, Echenique and Fryer 2007). Massey and Denton (1988) provides a survey of other measures meant to encapsulate various qualitative aspects of segregation, and motivates our decision to capture segregation by measuring exposure.

${ }^{19}$ This theoretical measure of experienced isolation is very related to Wong and Shaw's (2011) activity space approach. Both consider the individual-level exposure of one group to another based on the share of individuals in locations of activity. Instead of theorizing in terms of discrete locations, we depart from their approach and consider the idealized continuous set of locations. With an abuse of notation, one can think of $\int_{t=0}^{1} l(i, t)$ as the activity space for individual $i$.
} 
weight for individual $i$ to be

$$
\lambda_{i}=\frac{N_{c(i)}}{\tilde{N}_{c(i)}}
$$

where $N_{c}$ is the census population of tract $c$ and $\tilde{N}_{c}$ is the number of devices in our sample with home locations in tract $c$.

Combining these assumptions, we form an estimator of $S_{i}$ as follows. First, we form a leave-out estimate of $\bar{s}_{l}$ :

$$
\hat{s}_{l}^{-i}=\frac{\sum_{j \in \mathcal{P}_{l}^{-i} \cap W} \lambda_{j}}{\sum_{j \in \mathcal{P}_{l}^{-i}} \lambda_{j}},
$$

where $\mathcal{P}_{l}^{-i}$ is the set of pings associated with individuals other than $i$ who visit location $l$ and we abuse notation by letting $\lambda_{j}$ denote the weight of the individual associated with ping $j$. We omit visits by $i$ from this measure to avoid a severe small-sample bias that can arise when some locations have a small number of observed visits (Cortese et al. 1976; Carrington and Troske 1997; Gentzkow et al. 2019). Second, we estimate $\bar{S}_{i}$ by

$$
\hat{S}_{i}=\frac{1}{\left|\mathcal{P}_{i}\right|} \sum_{j \in \mathcal{P}_{i}} \hat{s}_{l(j)}^{-i},
$$

where $\mathcal{P}_{i}$ is the set of pings associated with $i$ and $l(j)$ is the location of ping $j$.

Finally, we estimate experienced isolation by

$$
\hat{E} I_{a}=\frac{1}{\left|W_{a}\right|} \sum_{i \in W} \lambda_{i} \hat{S}_{i}-\frac{1}{\left|N W_{a}\right|} \sum_{i \in N W} \lambda_{i} \hat{S}_{i} .
$$

We estimate residential isolation as

$$
\hat{R I_{a}}=\frac{1}{\left|W_{a}\right|} \sum_{i \in W_{a}} \lambda_{i} \hat{r}_{c(i)}-\frac{1}{\left|N W_{a}\right|} \sum_{i \in N W_{a}} \lambda_{i} \hat{r}_{c(i)}
$$

where $\hat{r}_{c}$ is the share of devices in our sample with home census tract $c$ that are WDs. This differs from the residential isolation measure typically reported in the literature because the types we consider are WDs and NWDs rather than white and black individuals and because we infer $\hat{r}_{c}$ from our device data rather than census data.

\subsection{Discussion}

Our measure of experienced isolation considers an individual to be exposed to another if they are in the same location at the same time. This is what allows us to write equation 3 replacing the $e_{i}$ of equation 1 with the average of $s(l, t)$ across space and time. The set of people that contribute to an individual's exposure is, as discussed in the introduction, quite different from the set of people with whom an individual actually interacts. To the extent that we view actual interactions as the true object of interest, our measure can be seen as an approximation which significantly improves on residential measures but may still over- or understate isolation to the extent that interactions within different geohash $7 \mathrm{~s}$ are relatively more or less segregated. In Section 5 we decompose isolation into features of a city like schools, churches, and restauraunts/bars to help inform the kinds of interactions implied by physical presence in the same geographic space.

In our empirical analysis, we define the types $W$ and $N W$ to be WDs and NWDs-devices from majority white and non-white home geohash7s — rather than white and non-white individuals. This is a departure from prior literature on residential segregation, where the assumption of uniform exposure within neighborhoods makes it possible to compute segregation based on individual race (using aggregate race shares measured in census data). 
Therefore, the target of our estimation is subtly different than the standard target. To gain some intuition for the difference, note that individual geohash7s are perfectly segregated between WDs and NWDs by construction, whereas they are less than perfectly segregated by individual race. As noted in Section 2.4, the median WD lives in a home geohash7 which is 89 percent rather than 100 percent white, and the median NWD lives in a home geohash7 which is 78 percent rather than 100 percent non-white. We show below that this leads residential isolation between WDs and NWDs to be higher than between individual whites and non-whites. While the true level of segregation under our definition may be different, we expect the qualitative patterns we emphasize-e.g., the comparison of residential to experienced segregation — to be robust across alternative definitions.

As support for this, we report in Appendix Section S3 results using an alternative strategy where we impute race stochastically at the individual device level based on the composition of a home geohash7. This has the advantage of bringing our target concept closer to that in the prior literature. It has the disadvantage of introducing measurement error in the measure of a device's type that could create a downward bias in experienced segregation estimates 20 While this alternative does change the level of segregation as expected, we confirm that our main qualitative conclusions are indeed robust.

\section{Main Results}

Figure 1 shows estimated experienced and residential isolation for all MSAs in our sample ${ }^{21}$ Two key facts are immediately apparent from these maps. First, experienced isolation is lower than residential isolation in large sections of the country. Second, the two measures are correlated across space, with both tending to be higher in the South, the Rust Belt, and in major cities, and tending to be lower in the upper Midwest and Northwest.

Figure 2 compares the two measures more directly, plotting experienced isolation against residential isolation. Experienced isolation is lower than residential isolation where residential isolation is high and higher than residential isolation where residential isolation is low. MSAs in the former category, however, account for the vast majority of the country's population, including all 15 of the most populous MSAs, with 87.9 percent of people living in MSAs where experienced isolation is less than residential isolation. The population-weighted average experienced isolation across all MSAs is 0.46 , compared to average residential isolation of 0.61 . The 10th and 90th percentiles of experienced isolation are 0.37 and 0.53 , compared to 0.34 and 0.78 for residential isolation. This figure also confirms that experienced and residential isolation are highly correlated across MSAs, with a Pearson correlation coefficient of 0.864 and a Spearman rank correlation coefficient of 0.84. Among the 20 most populous MSAs, the ratio of experienced isolation to residential isolation is lowest $(\sim 0.6)$ in San Francisco-Oakland-Fremont, CA and Los Angeles, CA and highest $(\sim 0.8)$ in Atlanta, GA, and Riverside, CA.

To describe the factors that correlate with lower experienced segregation, we regress experienced isolation on observed MSA characteristics controlling for fifteen equal-sized bins of residential isolation. We focus on populationweighted univariate relationships, including a single observed characteristic in each case ${ }^{22}$ We emphasize that these are purely descriptive correlations and need not imply anything about the causes or effects of segregation.

Figure 3 shows the results. Each panel plots residuals of experienced isolation against residuals of a given MSA characteristic after partialing out the residential isolation controls. Experienced isolation is relatively lower in MSAs with higher population density and more public transit use. This is consistent with the fact that in dense areas residents from different neighborhoods are less separated by physical space, and may reflect the role of urban amenities such as parks and public facilities in facilitating diverse interactions (Jacobs 1961). Experiences are also relatively

\footnotetext{
${ }^{20}$ The random imputation strategy is equivalent to assuming that movement patterns are independent of individual race conditional on home geohash7. In simulations, we find that this tends to lead to a downward bias in estimates of experienced segregation.

${ }^{21}$ Appendix Figure $\mathrm{S9}$ presents a map with the difference between experienced and residential isolation for each MSA. Appendix Tables S5. S7 report both experienced and residential isolation for each MSA.

${ }^{22}$ Appendix Table S8 shows similar results in regressions that are unweighted but subset to the top 50, 100, and 200 most populous MSAs.
} 
less isolated in MSAs with higher income, more education, and lower unemployment. This could reflect a number of forces including the role of social capital in reducing segregation (Putnam 2000). Experienced isolation is relatively lower where populations are younger, possibly reflecting the importance of schools and workplaces in reducing segregation. Finally, relative experienced isolation is negatively correlated with Chetty et al.'s (2014) measures of income mobility for both black and white individuals, consistent with both diverse interactions increasing mobility and with areas that facilitate opportunity also promoting diverse interactions.

\section{Decomposing Experienced Isolation}

\subsection{By Time}

We first ask how experienced isolation varies over hours of the day. To do this, we restrict both exposures and the set of devices to all those that occur in a specific hour according to the MSA's local time zone. Exposures are only estimated in geohash7s that are visited by devices that ping within that hour. For example, experienced isolation for 10 a.m. restricts our sample to pings that occur between 10 a.m. and 11 a.m. local time. After restricting the set of pings and devices, the estimation of experienced isolation is identical to our baseline measure.

Figure 4 plots experienced isolation over the course of the day, scaled relative to the level of residential isolation. The figure highlights the 10 most populous MSAs. The results are intuitive: Experienced isolation is lowest in the middle of the day as people move around and highest late at night as people withdraw into their homes. The ratio mostly differs in level between MSAs and almost all MSAs share the same time profile.

\subsection{By Location}

We next decompose experienced isolation by location. Much like restricting to pings within an hour, we restrict to pings that occur within a set of geohash7s of a particular type ${ }^{23}$ These results are shown in Figure 5. The leftmost point in the plot shows the average of our baseline measure of experienced isolation across MSAs, which includes all locations in our sample. The error bars in the plot indicate \pm 1 standard deviation of the measure across MSAs.

The next two points in the figure show experienced isolation for locations within vs. outside of home census tracts. The results show that experienced isolation within home tracts ( 0.63 on average across MSAs) is higher than overall experienced isolation ( 0.46 on average), and actually higher than residential isolation ( 0.61 on average) ${ }^{24}$ As discussed above, this result is not mechanical: experienced isolation within the home tract could differ from residential isolation in either direction, both because within-tract exposure is not uniform and because it includes visitors who live outside the home tract. In contrast, experienced isolation outside of home tracts is much lower, with an average of 0.21 across MSAs. Thus, time spent away from home is the key force reducing segregation relative to what the standard residential measure would suggest.

Figure 5 summarizes the differences in experienced isolation for specific categories of features 25 The baseline category contains all features, as well as time spent at home. Average experienced isolation in outdoor spaces like parks, gardens, sports fields and playgrounds is only 50.3 percent of mean baseline isolation, and commercial establishments like restaurants and bars and retail stores have experienced isolation that is only 43.5 and 47.8 percent of baseline isolation respectively. Isolation is among its lowest in places of entertainment like theaters ( 24.3 percent of baseline) and accommodations like hotels (24.6 percent of baseline). Appendix Table S9 shows summary statistics for experienced isolation across a wider set of feature types.

\subsection{By Race}

Finally, we can decompose the differences in exposure that underlie the isolation index between WDs and NWDs. Experienced isolation is the difference between these groups in average exposure $E[s(l, t)]$. We ask how the experi-

\footnotetext{
${ }^{23}$ If an individual never visits a geohash7 of the type in question they are dropped from the sample.

${ }^{24}$ Appendix Figure $\mathrm{S} 10$ depicts experienced isolation within and outside home tracts.

${ }^{25}$ Appendix Figure $\overline{S 13}$ depicts ping activity across features by WD/NWD designation.
} 
enced exposure relative to residential exposure differs by group. The results, which we present in Appendix Figures S11 and S12, show that the difference between experienced and residential exposure is relatively small for WDs and much larger for NWDs. It also shows that NWDs' experienced exposure varies much more across MSAs and across

different feature types. This suggests that factors which reduce segregation away from home may have a particularly large impact on the experiences of NWDs.

\section{Robustness}

The Online Appendix reports a number of additional specifications probing the robustness of our main result. We provide detail on these specifications in Online Appendix $\mathrm{S} 2$ and show the results in Online Appendix Table S10. They show that our main qualitative conclusions are robust to: (i) excluding pings that are likely to occur while devices are commuting or traveling; (ii) using alternative sources of demographic data; (iii) excluding devices with home locations outside the MSA; (iv) dropping the top 5 percent of devices in terms of number of pings per day; (v) excluding pings occurring between midnight and 6 a.m.; (vi) using only the first ping emitted by a device in a given hour (so as to avoid over-weighting hours with frequent pings). The final result in this table shows that we would over-estimate experienced segregation if we used a naive estimator rather than the leave-out correction in equation (5).

\section{Conclusion}

The extent to which members of different groups are able to see, meet, and interact with one another can profoundly shape economic and social outcomes. Standard isolation indices capture such patterns under the assumption that people are uniformly exposed to others in their neighborhoods of residence. Our measure of experienced isolation builds on the activity space literature to relax this assumption and leverage novel location data to describe the exposures people actually experience as they move around over the course of their days.

We find that the exposure to diverse others that people actually experience is substantially greater than residential measures would suggest. People spend substantial time away from their home neighborhoods, and when they do they are much more likely to encounter diverse others than they would at home. Commercial places like restaurants and retail shops are a particularly strong force pulling against segregation, while local amenities such as churches and schools tend to remain more segregated. One implication is that public goods that are tied to residential boundaries may deserve particular attention in efforts to combat segregation.

While experienced and residential segregation are highly correlated across cities, the gap between them varies systematically, with relatively less experienced isolation in cities that are denser, wealthier, and more educated, that have greater use of public transport, and where income mobility is higher. These correlations do not allow us to draw any direct conclusions about either the causes or consequences of segregation, but they point toward factors that will be especially fruitful for subsequent research to investigate. 


\section{References}

Allcott, H., R. Diamond, J.P. Dubé, J. Handbury, I. Rahkovsky, M. Schnell. 2019. "Food Deserts and the Causes of Nutritional Inequality.” The Quarterly Journal of Economics 134(4):1793-1844.

Blattman, C., D. P. Green, D. Ortega and S. Tobon. 2019."Place Based Interventions at Scale: The Direct and Spillover Effects of Policing and City Services on Crime.” Working Paper.

Browning, CR., CA. Calder, B. Soller, AL. Jackson, J. Dirlam. 2017. "Ecological networks and neighborhood social organization.” American Joural of Sociology. 122:1939-1988.

Caetano, G. and V. Maheshri. 2019. "Gender Segregation within Neighborhoods." Regional Science and Urban Economics 77:253-263.

Cagney, KA., E. York Cornwell, AW. Goldman, L. Cai. 2020. "Urban mobility and activity space". Annu. Rev Sociol. 46:623-648.

Card, D., A. Mas and J. Rothstein. 2008. “Tipping and the Dynamics of Segregation.” The Quarterly Journal of Economics 123(1):177-218.

Carrington, William J., Kenneth R. Troske. 1997. “On Measuring Segregation in Samples with Small Units.” Journal of Business \& Economic Statistics 15(4):402-409.

Chen, K.M., K. Haggag, D. Pope and R. Rohla. 2019. "Racial Disparities in Voting Wait Times: Evidence from Smartphone Data.” NBER Working Paper No. 26487.

Chen, K. M. and R. Rohla. 2018. "The Effect of Partisanship and Political Advertising on Close Family Ties." Science 360(6392):1020-1024.

Chetty, R., N. Hendren, P. Kline, E. Saez. 2014. "Where is the land of Opportunity? The Geography of Intergenerational Mobility in the United States.” The Quarterly Journal of Economics 129(4):1553-1623.

Chetty, R. and N. Hendren, 2018a. "The Impacts of Neighborhoods on Intergenerational Mobility I: Childhood Exposure Effects.” The Quarterly Journal of Economics 133(3):1107-1162.

Chetty, R. and N. Hendren, 2018b. "The Impacts of Neighborhoods on Intergenerational Mobility II: County-Level Estimates." The Quarterly Journal of Economics 133(3):1163-1228.

Chetty, R., N. Hendren and L. F. Katz. 2016. "The Effects of Exposure to Better Neighborhoods on Children: New Evidence from the Moving to Opportunity Experiment.” The American Economic Review 106 (4):855-902.

Chetty, R., N. Hendren, M. R. Jones and S. R. Porter. 2020. "Race and Economic Opportunity in the United States: An Intergenerational Perspective.” The Quarterly Journal of Economics 135(2):711-783.

Cortese, C. F., R. F. Falk and J. K. Cohen. 1976. "Further Considerations on the Methodological Analysis of Segregation Indices.” American Sociological Review 41(4):630-37.

Cowgill, D. O. and M. S. Cowgill. 1951. "An Index of Segregation Based on Block Statistics.” American Sociological Review 16:825-31.

Cutler, D. M. and E. L. Glaeser. 1997. “Are Ghettos Good or Bad?” The Quarterly Journal of Economics 112(3):827-72.

Cutler, D. M., E. L. Glaeser and J. L. Vigdor. 1999. “The Rise and Decline of the American Ghetto.” The Journal of Political Economy 107(3):455-506.

Davis, D. R., J. I. Dingel, J. Monras and E. Morales. 2019. “How Segregated Is Urban Consumption?” Journal of Political Economy 127(4).

Duncan, O. D. and B. Duncan. 1955. "A Methodological Analysis of Segregation Indexes." American Sociological Review 20(2):210-17. 
Echenique, F. and R. G. Fryer. 2007. “A Measure of Segregation Based on Social Interactions.” The Quarterly Journal of Economics 122(2):441-85.

Gentzkow, M. and J. M. Shapiro. 2011."Ideological Segregation Online and Offline.” The Quarterly Journal of Economics 126(4):1799-1839.

Gentzkow, M., J. M. Shapiro, and M. Taddy. 2019.“Measuring Group Differences in High-Dimensional Dhoices: Method and Application to Congressional Speech." Econometrica 87(4):1307-1340.

Glaeser, E. L., S.D. Kominers, M. Luca and N. Naik. 2018. "Big Data And Big Citites: The Promises and Limitations of Improved Measures of Urban Life.” Economic Inquery 56:114-137.

Iceland, J., D. H. Weinberg and E. Steinmetz. 2002."Racial and Ethnic Residential Segregation in the United States: 1980-2000." Census 2000 Special Reports.

Jacobs, Jane. 1961. The Death and Life of Great American Cities. New York : Vintage Books.

Jahn, J. A. 1950. "The Measurement of Ecological Segregation: Derivation of an Index Based on the Criterion of Reproducibility.” American Sociological Review 15:101-104.

Jones, M., AR. Pebley. 2014. "Redefining neighborhoods using common destinations: Social characteristics of activity spaces and home census tracts compared.” Demography 51:727-752.

Lee, LJZ., Shu. En, L. Shen. 2021. "Segregation across neighborhoods in a small city.” Asia Compet. Inst. Res. Pap. Ser.

Massey, D. S. and N. A. Denton. 1988. "The Dimensions of Residential Segregation.” Social Forces. A Scientific Medium of Social Study and Interpretation 67(2):281-315.

Massey, D. S. and N. A. Denton. 1993. American Apartheid: Segregation and the Making of the Underclass. Harvard University Press.

Moore RT, Reeves A. 2020. "Defining racial and ethnic context with geolocation data. Political Science Research and Methods." 8:780-794.

Park, Y.M. and M. Kwan. 2018. "Beyond Residential Segregation: A Spatiotemporal Approach to Examining Multi-Contextual Segregation.” Computers, Environment and Urban Systems 71:C.

Phillips, N.E., B.L. Levy, R.J. Sampson, M.L. Small and R.Q. Wang. 2021. "The Social Integration of American Cities: Network Measures of Connectedness Based on Everyday Mobility across Neighborhoods.” Social Methods \& Research 50:1110-1149.

Putnam, R.D. 2000. Bowling Alone: The Collapse and Revival of American Community. New York: Simon \& Schuster.

Sampson, RJ., BL. Levy. 2020. "Beyond residential segregation: mobility-based connectedness and rates of violence in large cities." Race social problems 12:77-86.

Shelton, T., A. Poorthuis, M. Zook. 2015. "Social media and the city: Rethinking urban socio-spatial inequality using user-generated geographic information" Landsc. Urban Planning 142:198-211.

Sunstein, C. R. 2002. Republic.com. Princeton University Press.

Taeuber, K. E. and A.F. Taeuber. 1965. Negroes in Cities: Residential Segregation and Neighborhood Change. Chicago. Aldine Pub. Co.

White, M. J. 1983. "The Measurement of Spatial Segregation. "American Journal of Sociology 88(5):1008-1018.

White, M. J. 1986. "Segregation and Diversity Measures in Population Distribution." Population Index 52 (2):198-221.

Wong, DW., SL. Shaw. 2011. “Measuring segregation: An activity space approach.” Joural of Geographical Systems 

13:127-145.

Zhang, X., J. Wang, MP. Kwan, Y. Chai. 2019. "Reside nearby, behave apart? activity-space-based segregation among residents of various types of housing in Beijing, China." Cities 88:166-180. 
Experienced isolation

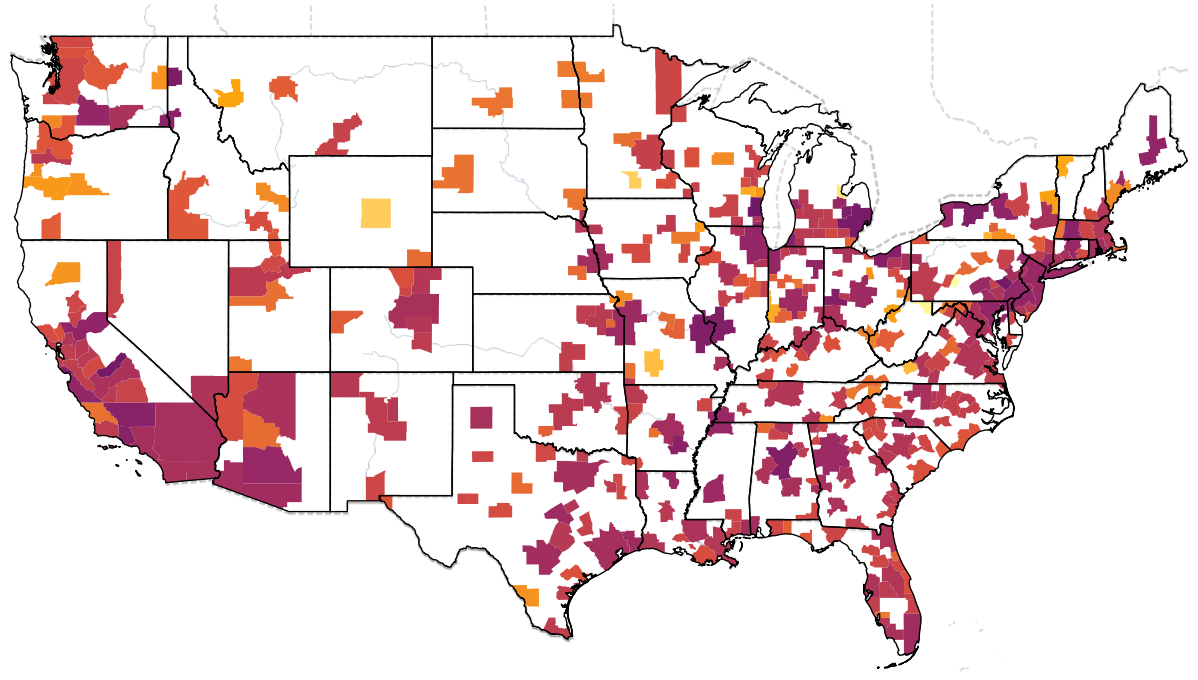

Residential isolation

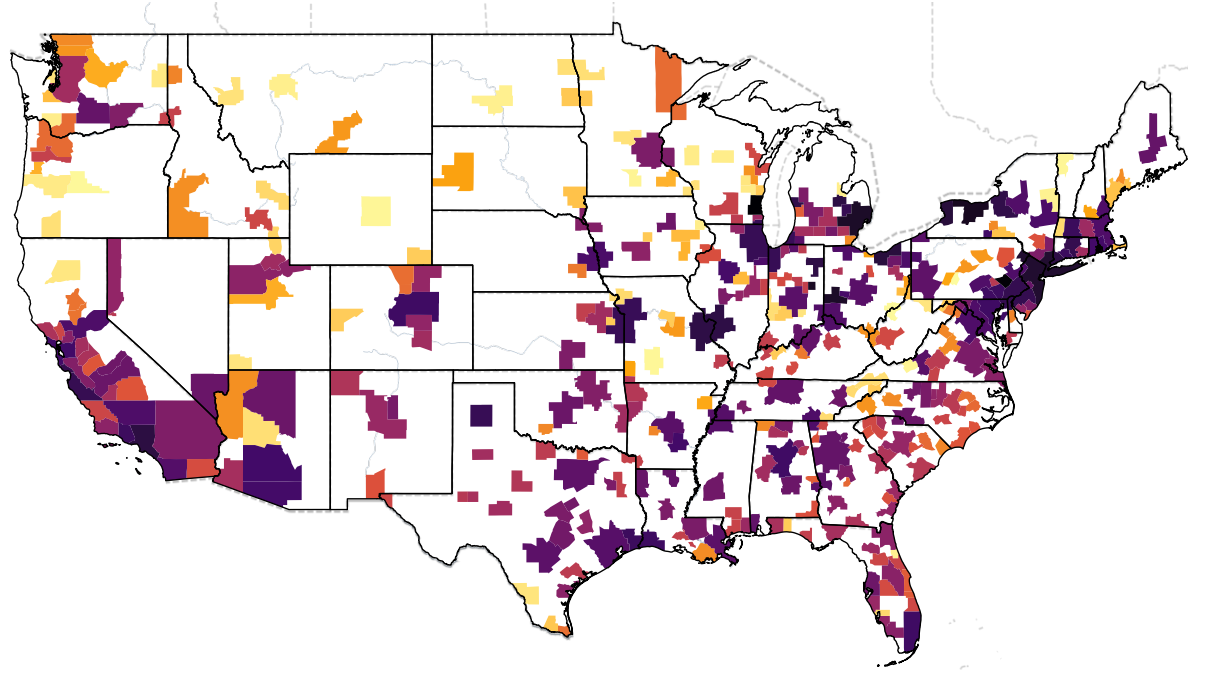

Isolation

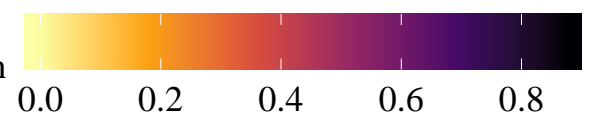

Figure 1: Experienced and Residential Isolation by MSA 


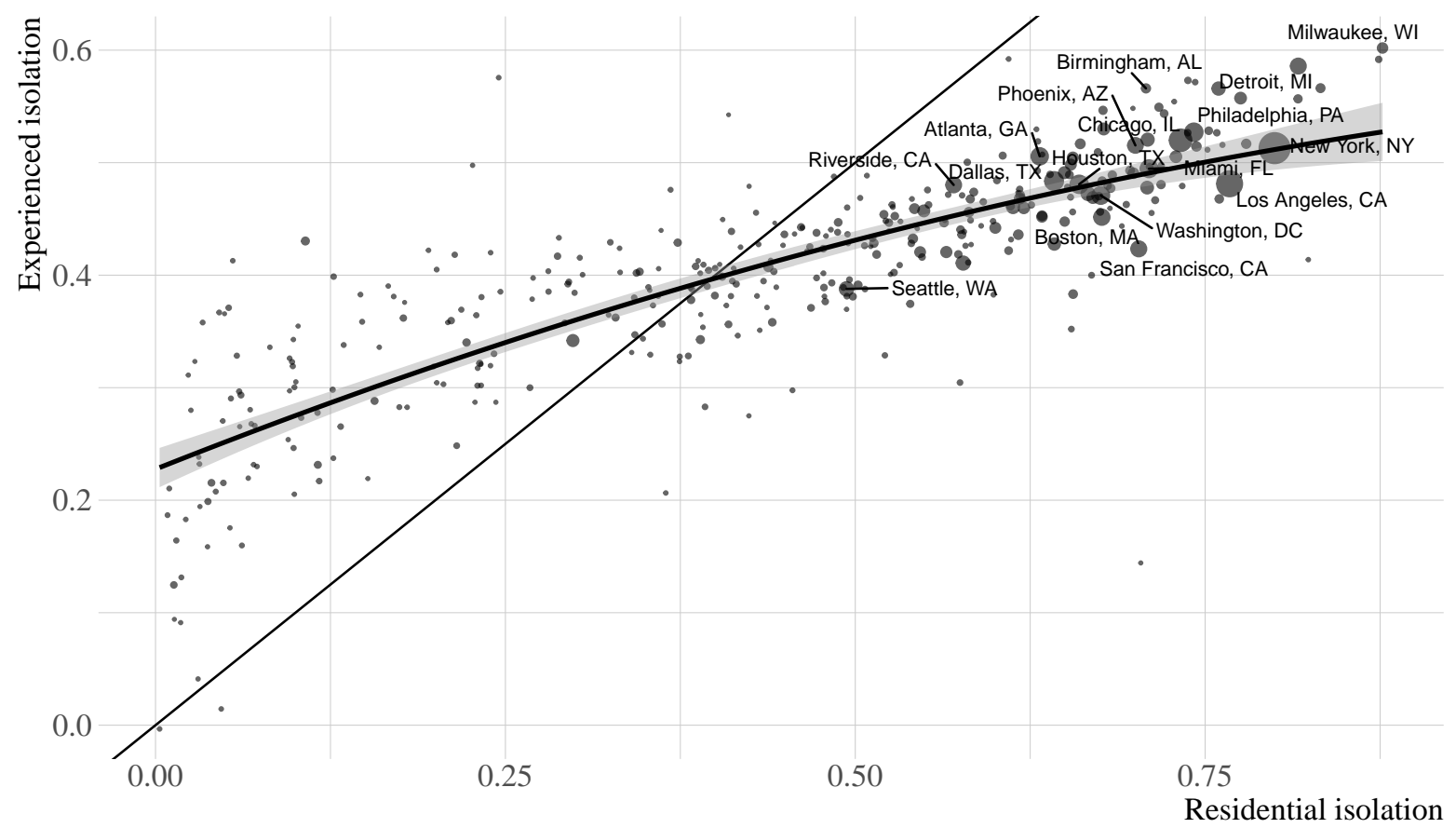

Figure 2: Experienced vs. Residential Isolation

Notes: Plot shows experienced and residential isolation for each MSA. The size of each point is proportional to the MSA's population. The labeled points designate the 15 most populous MSAs. We plot the 45 degree line and a local polynomial fit. 

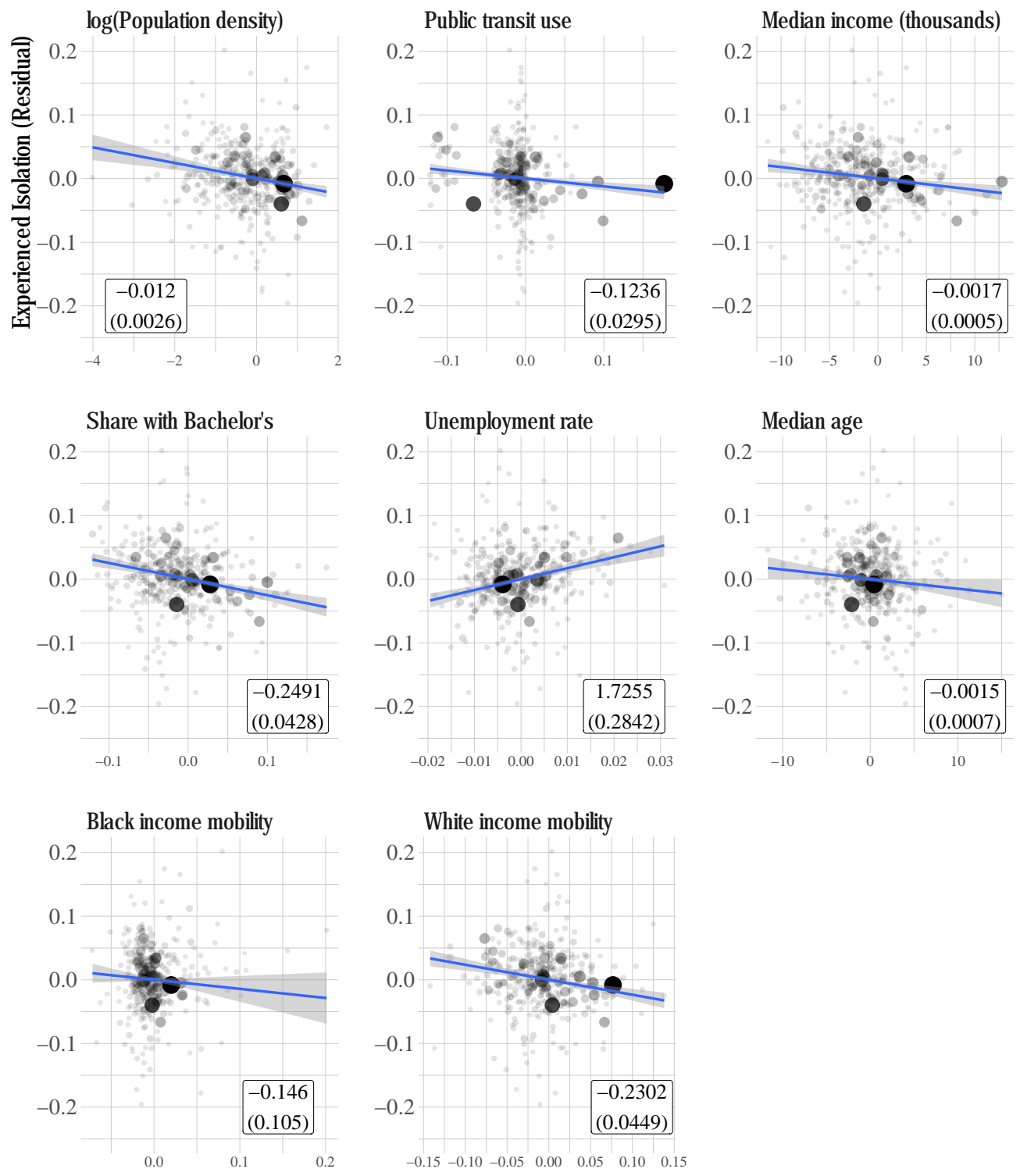

Figure 3: Residual Experienced Isolation and MSA Characteristics

Notes: On the y-axis, we plot the residual from a population weighted regression of experienced isolation on fifteen equal sized bins of residential isolation at the MSA level. The x-axis in each plot refers to the specified MSA characteristic. Each point refers to an MSA and is shaded and sized relative to total population. In the white box in the lower left corner, we show the coefficient and standard error from the population weighted regression of experienced isolation on the residential isolation bin fixed effects and the specified covariate. The blue line shows the population weighted linear fit. The share with bachelor's variable includes the percent of people in an MSA that have at least a bachelor's degree. The black and white income measures average Chetty et al.'s (2020) county estimates (pooled by race) of the share of individuals born in the 25th percentile of the income distribution who make it to the top quintile. Public transit use is the share of the working population that uses public transport to get to work. 


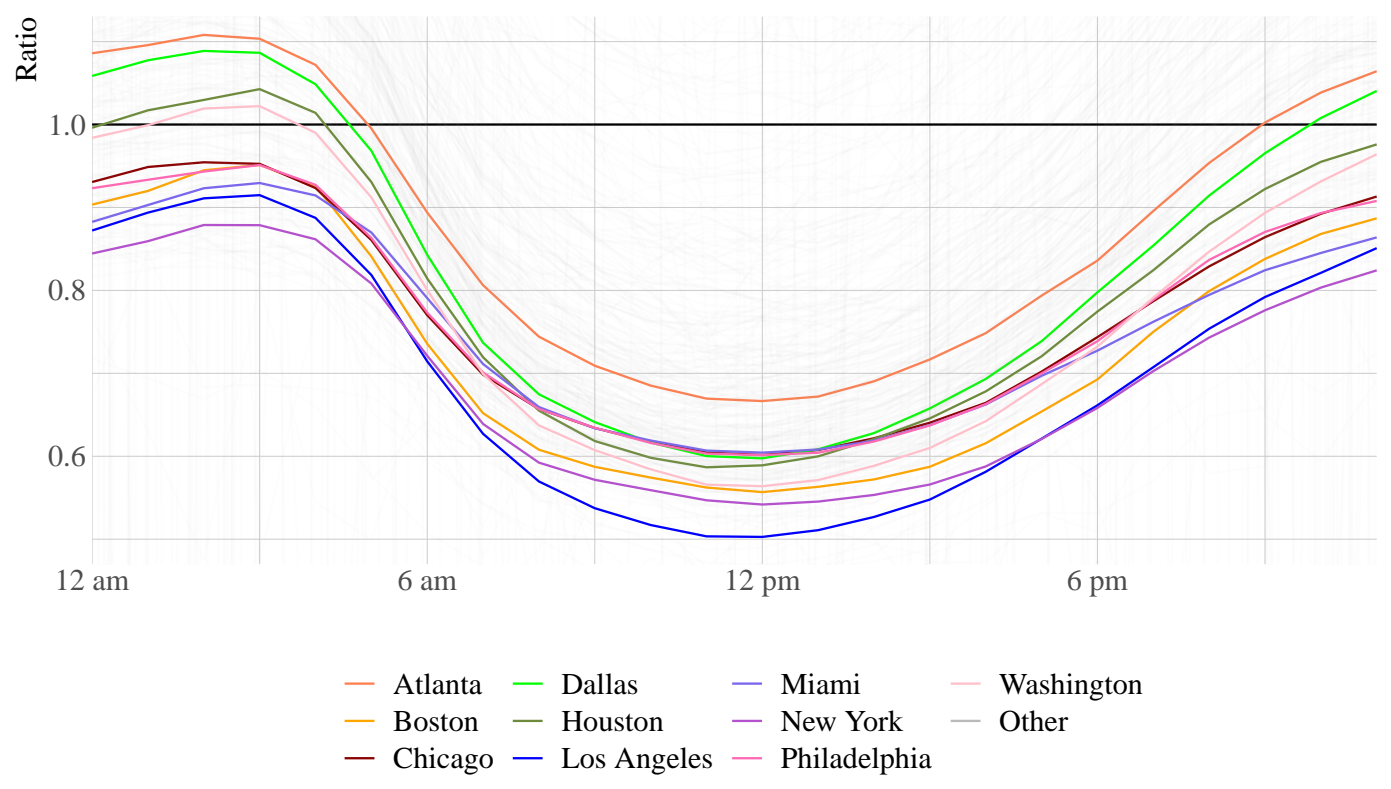

Figure 4: Experienced Isolation Relative to Baseline by Time of Day

Notes: We plot the ratio of experienced to residential isolation in each hour of the day, highlighting the 10 most populous MSAs. Note that isolation can only be calculated for the devices active in a given hour, so the sample does change for each hour specification. 


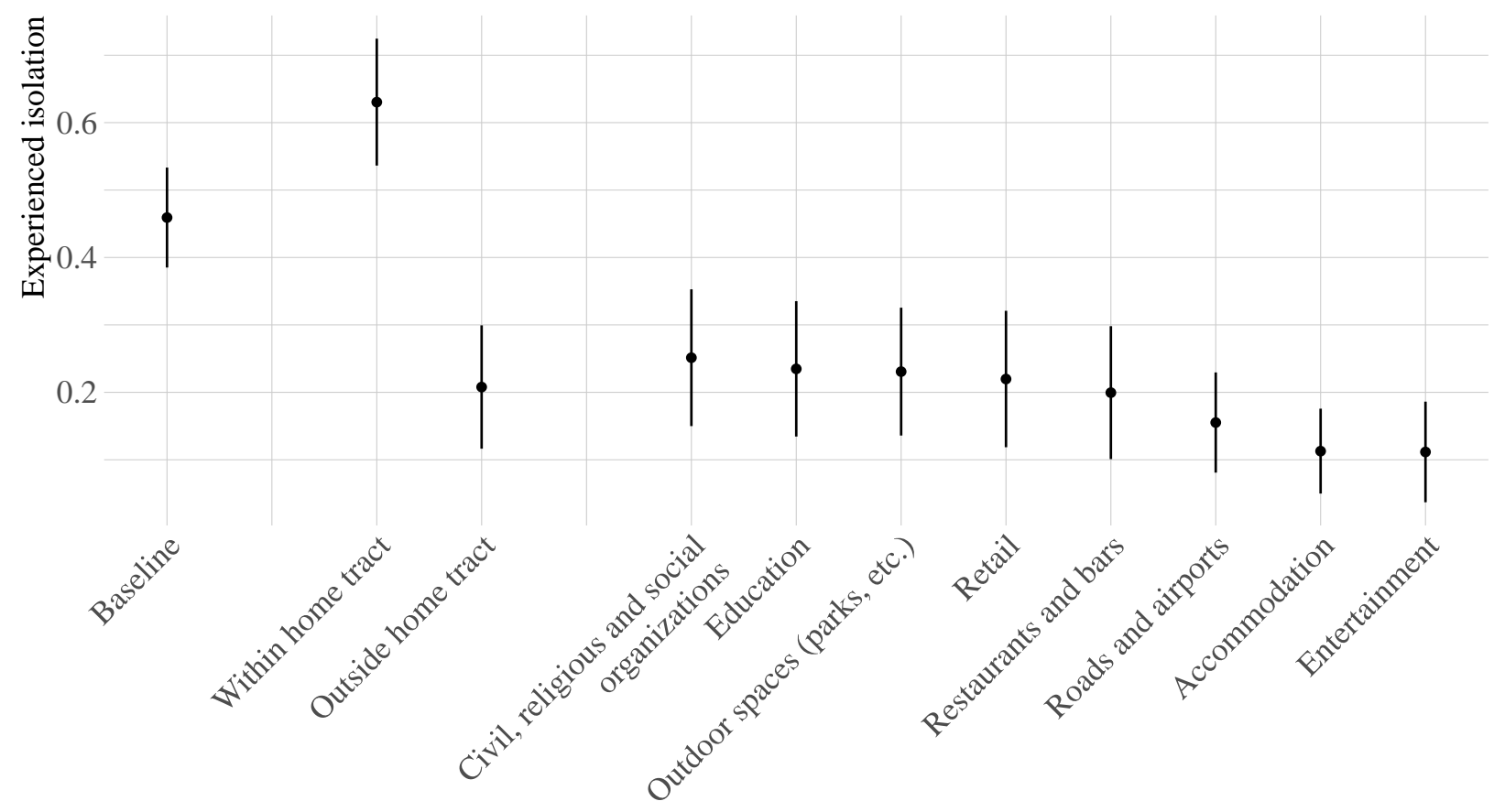

Figure 5: Experienced Isolation Relative to Baseline by Location

Notes: We plot the population weighted mean experienced isolation in a particular feature and compare with our baseline measure. Error bars show \pm the population weighted standard deviation of experienced isolation across MSAs. 


\title{
Supporting Information (SI): Estimating Experienced Racial Segregation in U.S. Cities Using Large-Scale GPS Data
}

\author{
Susan Athey, Stanford University and NBER \\ Billy Ferguson, Stanford University \\ Matthew Gentzkow, Stanford University and NBER \\ Tobias Schmidt
}

\section{S1 Defining Geographic Features}

\section{S1.1 InfoUSA}

We use data from InfoUSA to define the following features: (i) civil, religious, and social organizations; (ii) retail; (iii) restaurants and bars; (iv) accommodation; (v) entertainment; (vi) sports and recreation. We combine InfoUSA data with Open Street Maps data to define education features, as detailed in Appendix $\mathbf{S 1 . 2}$ below.

For each feature, InfoUSA provides latitude, longitude, and an 6-digit NAICS industry code. We focus on the top 334 NAICS codes in the data which together cover 95 percent of all establishments and assign them manually to our aggregated categories. The mapping between NAICS6 and categories is given in Table S1. We assign each feature to the geohash7 that contains its latitude-longitude pair.

\section{S1.2 OpenStreetMaps}

We use data from Open Street Maps (OSM) to define outdoor spaces and to supplement InfoUSA data in defining education features. OSM is an open source mapping project that defines geographic features and associates them with metadata tags. Whereas InfoUSA provides point locations for each feature, OSM defines two-dimensional polygons. These provide a more accurate representation for features like parks that occupy a large amount of space. As noted in the main text, we associate each OSM feature with all geohash7s that intersect its polygon.

We define features to be outdoor spaces if they are associated with the tags "leisure=park," "leisure=playground," "leisure=pitch," or "leisure=garden." We define features to be education-related if they are associated with the tags "amenity=school," "amenity=kindergarten," "amenity=university," or "amenity=college." We define geohash7s to contain education features if they are associated with such a feature in either InfoUSA or OSM.

\section{S1.3 Transportation Infrastructure}

We define transportation features by combining polygon data on primary and secondary roads from the US Census' TIGER database (U.S. Census Bureau 2017) with airport polygon data from OSM (polygons with the tag aeroway=aerodrome). We associate each such feature with all geohash7s that intersect its polygon.

\section{S2 Robustness}

Table $\mathrm{S} 10$ probes the robustness of our experienced isolation estimates. The first row repeats our baseline estimate. Each subsequent row reports a separate robustness check. For each, we report the mean, median, 5th, and 95th percentiles of estimated experienced isolation by MSA, as well as the correlation between that row's estimates and the baseline.

\section{S2.1 Excluding Transportation Infrastructure}

The first two robustness checks show how the results change if we exclude pings that are likely to come while people are in transit. People sharing the same space while commuting or traveling (e.g., driving next to another car on a highway) may be relatively unlikely to have meaningful interactions, and so it is interesting to know whether these 
observations play a large role in our conclusions. Figure S14 shows the frequency of pings across geohash7s in Birmingham, AL, confirming visually that a substantial amount of activity indeed occurs on roads 26

In row (2) of Table S10, we exclude all pings in geohashes we identify as containing roads or airports. In row (3) we exclude all pings that are part of a sequence suggesting the device is moving at more than 12 miles per hour ${ }^{27}$ In both cases experienced isolation rises, consistent with time in transit having lower than average isolation, but the difference is modest and the correlation with the baseline estimates is high.

\section{S2.2 Imputing Geohash7 Demographics from Individual Data}

Our baseline estimates impute geohash7 demographics from 2010 census data as described in Section 2.3. A weakness of this approach is that census blocks (the smallest units at which demographics are reported in the census) do not align exactly with geohash7s. An alternative approach is to impute geohash7 demographics from individual-level data sources where we observe individuals' exact addresses.

Row (4) of Table S10 reports estimates based on demographics imputed from an individual-level voter file provided by the company L2. These data provide home addresses of registered voters. The data include self-reported race in eight states that collect this as part of the registration process (Alabama, Florida, Georgia, Louisiana, North Carolina, South Carolina, Tennessee and Texas), and imputed race based on a proprietary algorithm elsewhere. We use the L2 category "European" as our measure of "white." We successfully match at least one L2 individual in $5,767,098$ of our 7,288,958 geohash7s. For the remaining geohash7s, we use our baseline imputation.

Row (5) reports estimates based on demographics imputed from individual-level data from the company Infutor. These data provide names, address, immigration status and demographics of around $80 \%$ of the adult population in the US. Race is defined in accordance with census categories and is imputed as described in Diamond et al. (2019). We exclude all individuals over the age of 80 and determine the home-geohash7 of each individual based on the last address they were registered at. We successfully match at least one Infutor individual in 5,079,532 of our 7,288,958 geohash7s. For the remaining geohash7s, we use our baseline imputation.

The results show that these alternative approaches lead to even lower estimates of experienced isolation, strengthening our main conclusion. The correlation with our baseline estimates remains high in both cases.

\section{S2.3 Alternative Temporal Weighting}

As discussed in Section 3.2, our baseline estimates rely on an assumption of uniform sampling. One way this may be violated is if some devices emit large numbers of pings at specific times when relevant apps are used heavily. In row (6) of Table S10, we partially address this possibility by only using the first ping emitted by a device in a given hour in a particular geohash7. This effectively gives equal weight to each device-geohash7-hour tuple in the data. We find experienced isolation is even lower in this specification, suggesting that non-random weighting may if anything lead us to understate the gap between residential and experienced isolation.

\section{S2.4 Alternative Unit of Exposure Defined at Hour-level}

In the baseline specification, all visitors to a particular geohash7 (regardless of time) contribute to the WD exposure of that geohash7. One could potentially be worried about segregated sorting over time within the same small geography. In Row (7) we present estimates from an hour-level specification where visitors to a geohash7 in a particular hour contribute to the WD expsoure of that geohash7-hour and visitors in other hours, even when visiting the same geohash7, are left out. Individual exposures are then averaged over geohash7-hours (instead of over "hour-blind" geohash7 exposures as in the baseline). The estimates in Row (7) suggest that accounting for this intertemporal sorting only slightly increases estimates of isolation relative to baseline. In Figure S15 we plot this alternative spec-

\footnotetext{
${ }^{26}$ Online Appendix Table $\mathrm{S} 11$ reports summary statistics for pings that maybe in transit.

${ }^{27}$ We take the sequence of timestamped latitudes and longitudes, compute the Haversine distance between successive pings in the sequence, and divide by the time difference to estimate device speed.
} 
ification of isolation that aggregates hour-level exposures against our baselines estimates to show that estimates are virtually the same across nearly every MSA.

It may seem contradictory that accounting for hour-level variation in geohash7 exposures does not change isolation estimates despite the dramatic variation in isolation over time shown in Figure 4; however, the combination of these results characterizes an interesting pattern of city-use. Since small geographies do not seem to experience demographic shifts in time, but individuals are more integrated in the middle of the day, it must be that there are areas in a city where people integrate and areas in which people are isolated. The isolation of a small geography is mostly determined by the geography and invariant to time-of-day. During mid-day indivdiuals visit the geohash7s that integrate, and at night, people return to isolated geohash7s.

\section{S2.5 Other Robustness Checks}

The final rows of Table $\mathrm{S10}$ consider other variations in the baseline sample. Row (8) drops pings from the sample devices whose home location is not in the same MSA as the ping, to give a sense of how out-of-town visitors influence the estimates. Row (9) drops the top 5 percent of devices in terms of number of pings per day, to address the possibility that such heavy users might have undue influence on the results. Row (10) excludes pings during late night hours from midnight to 6 a.m., to assess how much our results are influenced by the way we treat sleep time. None of these make a large difference to the estimates.

Finally, Row (11) shows how the results are impacted by the leave-out correction in equation (5). If we instead use the naive estimator that includes a device's own observations in the estimation of $\bar{s}_{l}$, we would over-estimate segregation. This is consistent with prior literature showing that this small-sample bias leads segregation to be overstated.

\section{S3 Alternative Definitions of Types}

Our baseline results measure segregation between WDs (devices from majority-white home geohash7s) and NWDs (devices from majority-non-white home geohash7s). In Table S12, we present results for racial segregation under alternative definitions of types.

In row (2), we change the definition of WD and NWD to depend on whether a home geohash7 is above or below the overall US share white (63 percent). Results are similar to our baseline estimates.

In the following rows, we shift focus to segregation between white and black home locations rather than white and non-white home locations. Row (3) defines the two types to be devices from home geohash7s with at least 50 percent white and at least 50 percent black respectively. Row (4) uses cutoffs of at least 70 percent white and at least 70 percent black respectively. Row (5) uses cutoffs of at least 90 percent white and at least 90 percent black respectively. Note that unlike in our baseline specification, some devices here fall into neither category and so are omitted from the analysis. Results are again similar to baseline, with somewhat lower estimates of both experienced and residential isolation when we use the more extreme cutoffs.

In rows (6)-(7), we present an approach to measuring experienced isolation by individual race. The differences between this and our baseline approach based on home geography are discussed in Section 3.3. We probabilistically impute individual race at the device level using the race shares in their home geohash7s (as measured by our baseline imputed demographics) as probabilities 28 This probabilistic imputation would correctly estimate segregation by individual race under the assumption that expected movement patterns are the same for devices of different races

\footnotetext{
${ }^{28}$ The direct imputation of race assigns to each device the home geohash7 share white and share nonwhite (or black) as constructed in Section 2.3 When we observe a device's ping in a geohash7, that device contributes the imputed share white to the exposure of that geohash7 instead of contributing a wholly white or nonwhite visit. Furthermore, when we construct the average exposure amongst white and nonwhite (or black) devices, we cannot simply take the average over devices of each type since each device is probabilistically assigned to both groups. Therefore, the average exposure of each group is calculated as a weighted average with the imputed home geohash 7 demographic shares as the weights.
} 
that come from the same geohash7. When this assumption is violated, we expect the systematic measurement error in imputed race to bias our experienced isolation measure downward.

Row (6) shows results where we probabilistically impute white and non-white race. Row (7) shows results where we probabilistically impute white and black race (allowing for an omitted category of devices that are neither black nor white). Consistent with expectations, estimates of both experienced and residential isolation fall significantly in these specifications. However, we find that experienced isolation is substantially lower than residential isolation and that the two are highly correlated across MSAs continue to hold.

\section{References}

Diamond, R., T. McQuade and F. Qian. 2019. "The Effects of Rent Control Expansion on Tenants, Landlords, and Inequality: Evidence from San Francisco.” American Economic Review 109(9):3365-94.

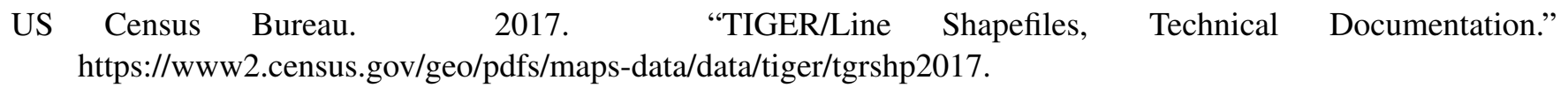




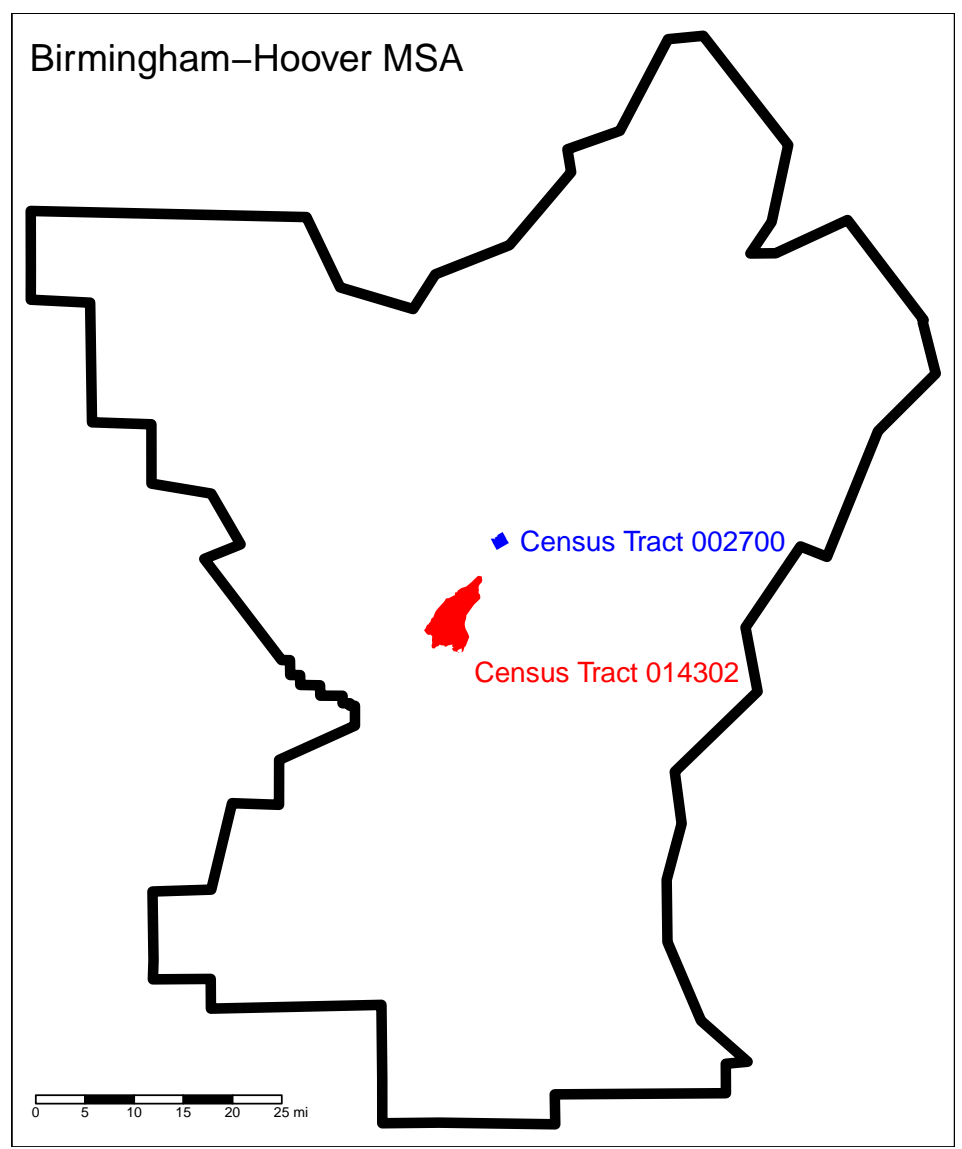

SI Figure S1: Birmingham-Hoover MSA and Census Tracts 002700 \& 014302

Notes: We depict the relative size of urban and rural tracts within the Birmingham-Hoover MSA, which is the final aggregate unit of analysis. 


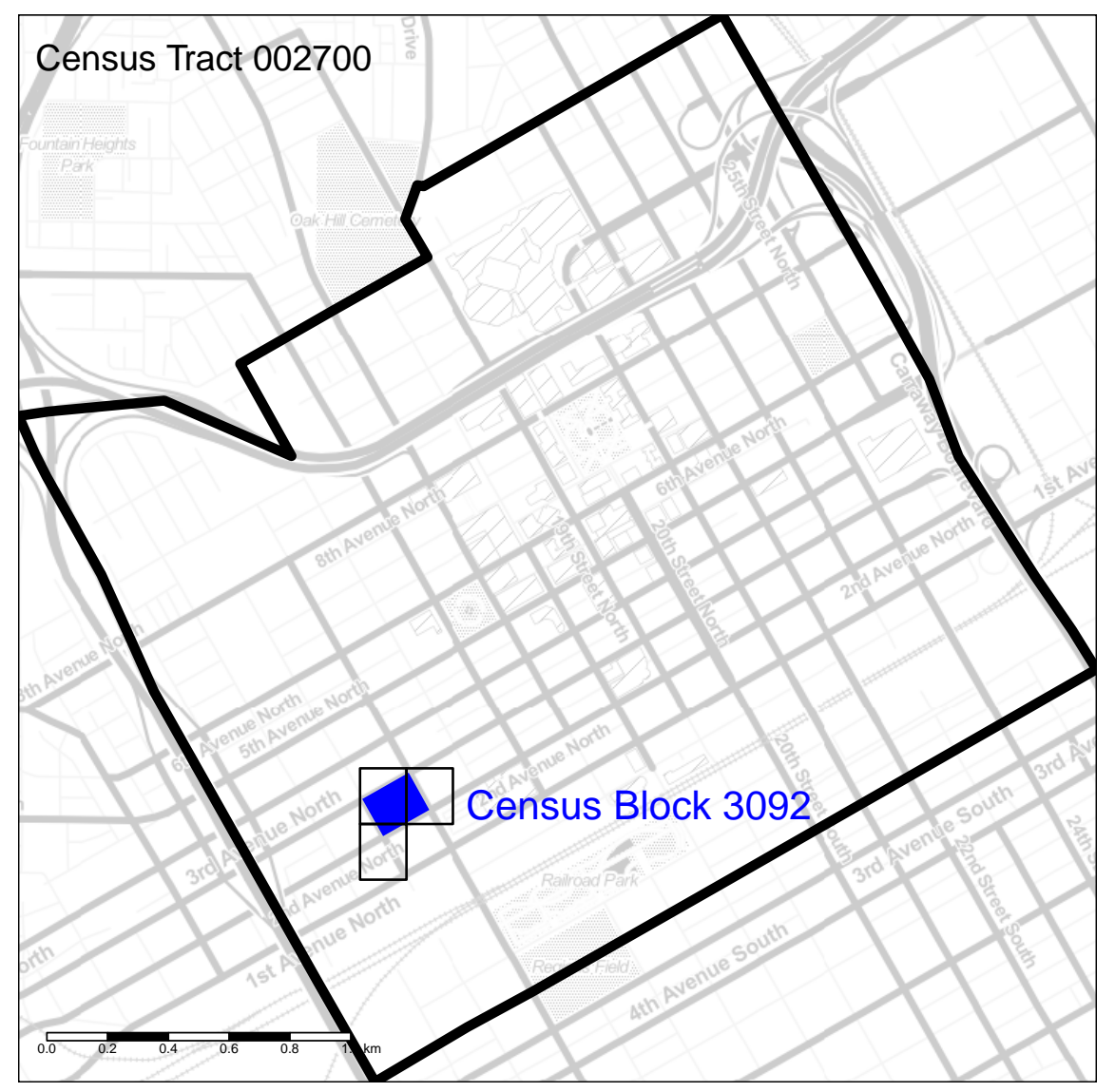

SI Figure S2: Relative Geographies in an Urban Center

Notes: This figure illustrates the relative size of census tracts / blocks and geohash7s in an urban area. The larger black outline depicts census tract 002700 in urban Birmingham, AL. The black grid consists of three geohash7s that overlap census block 3092. 


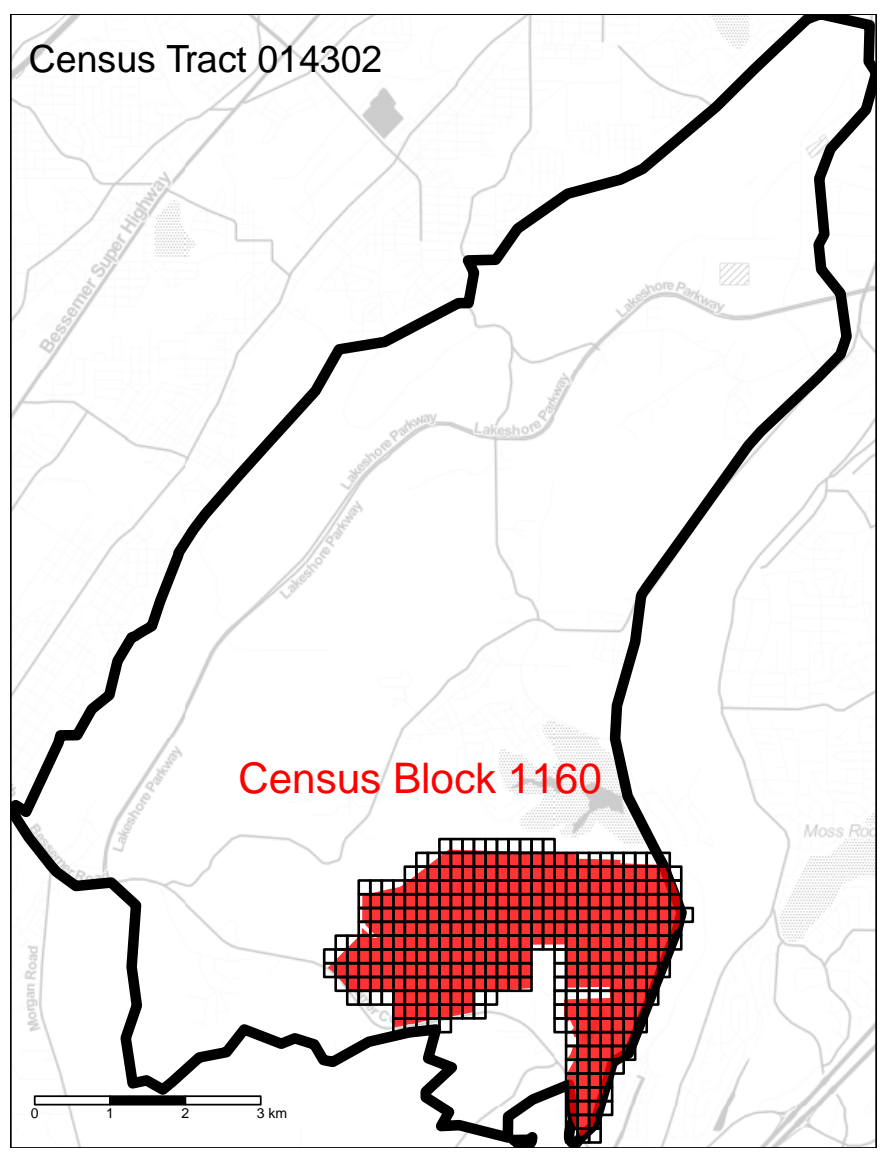

SI Figure S3: Relative Geographies in a Rural Setting

Notes: This figure illustrates the relative size of census tracts, blocks, and geohash7s in a rural area. The larger black outline depicts census tract 014302 in rural Birmingham, AL. The black grid consists of 367 geohash7s that overlap the census block 1160 . 


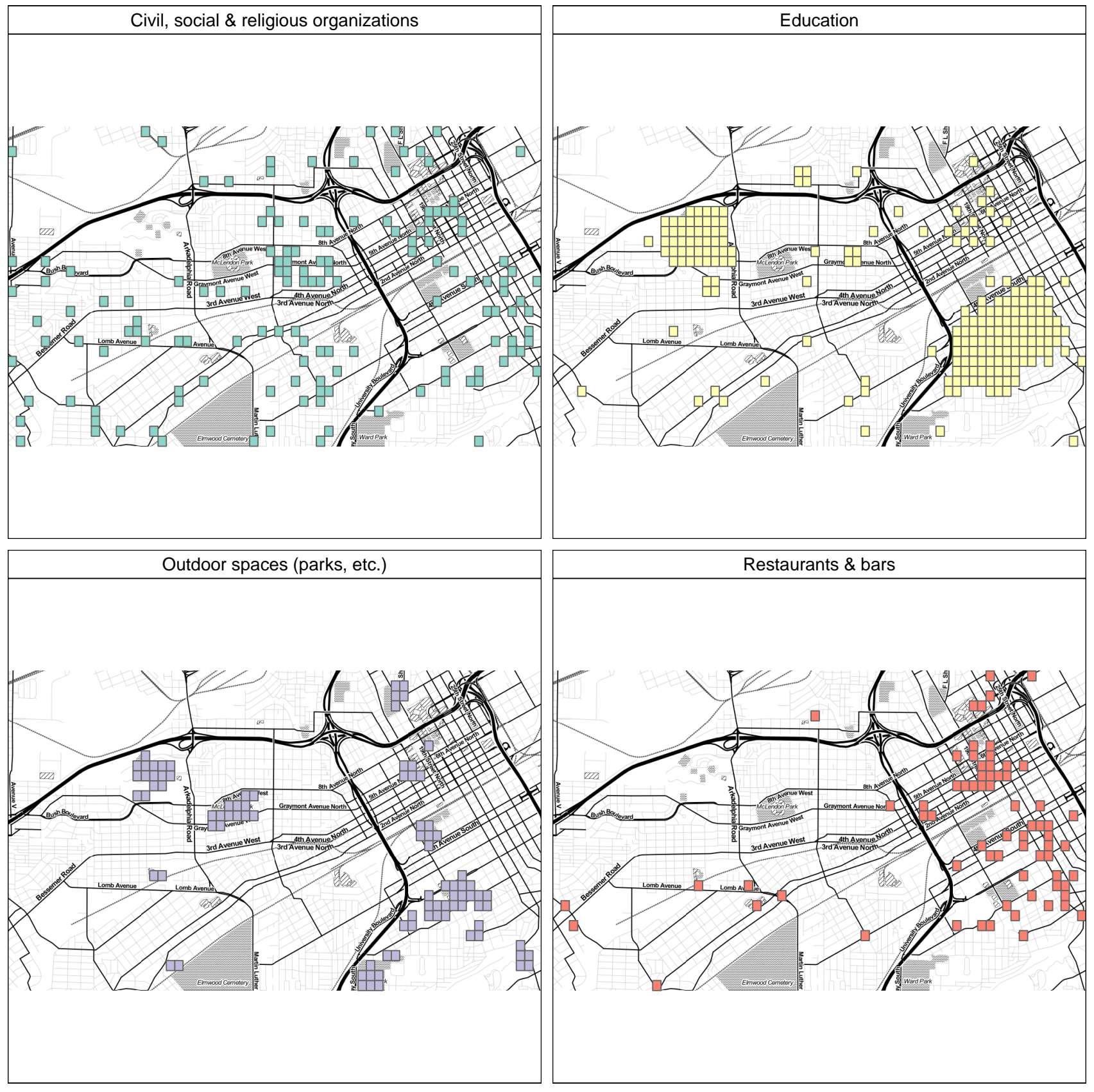

SI Figure S4: Features in Downtown Birmingham, AL

Notes: Figure highlights geohash7s that contain (i) civil, social, and religious institutions, (ii) educational instutitions, (iii) outdoor spaces, and (iv) restaurants and bars respectively, in downtown Birmingham, AL. A single geohash7 can contain multiple features. 


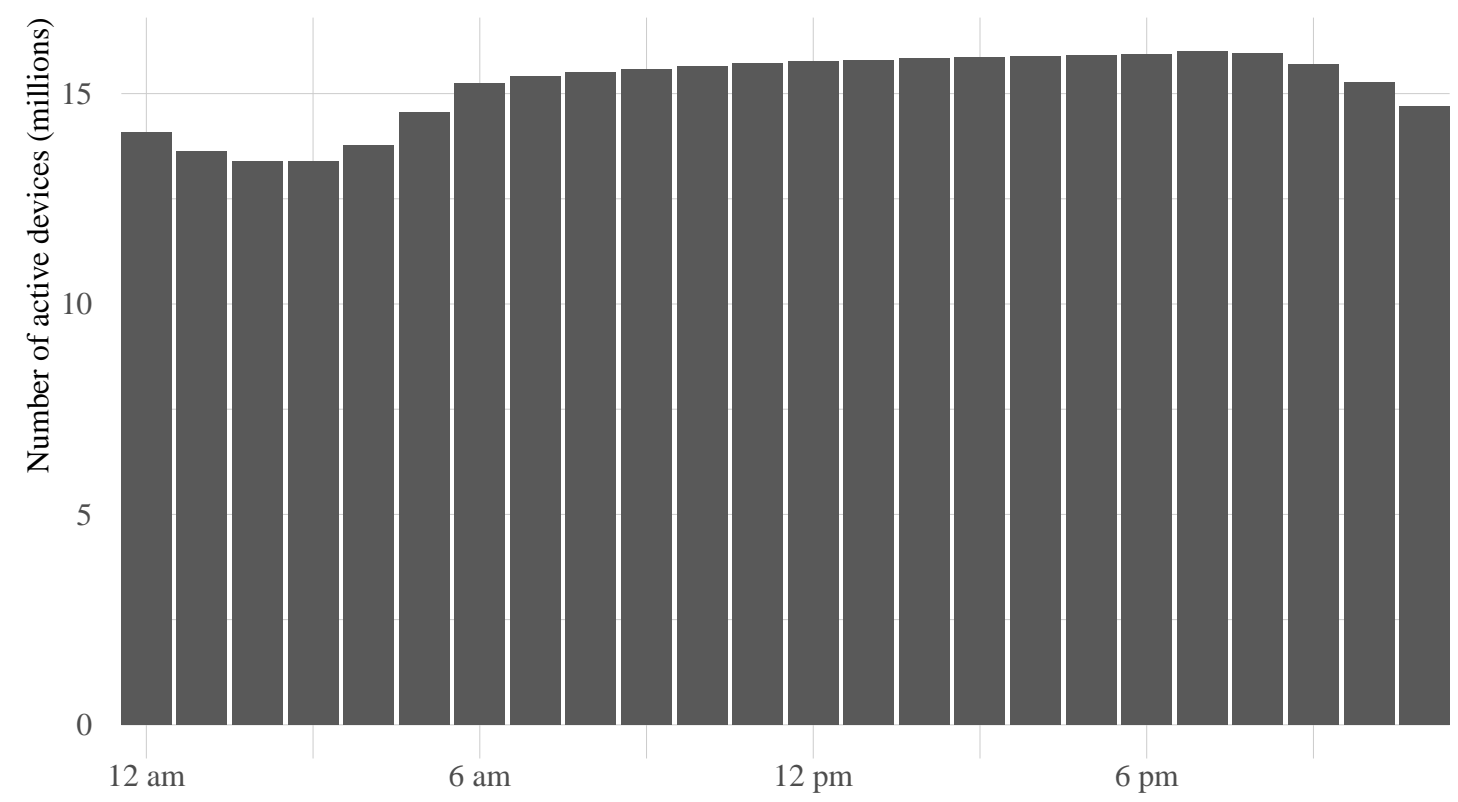

SI Figure S5: Number of Active Devices by Hour

Notes: We plot the number of active devices in millions by hour. A device is considered active if we ever observe at least one ping within the given hour. 


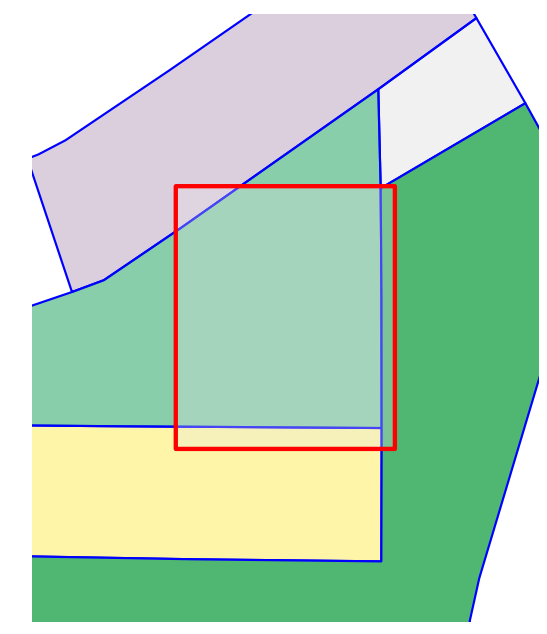

Percent white in census block

$40.0 \% 50.0 \% 60.0 \% 70.0 \%$

SI Figure S6: Matching Home Geohash7 to Blocks

Notes: Geohash7 djfq8cs in Jefferson county, AL is the rectangle outlined in red. Census blocks are the polygons outlined in blue. There are five census blocks overlapping the geohash7 which we color in relation to their share white. The grey census block is uninhabited. 


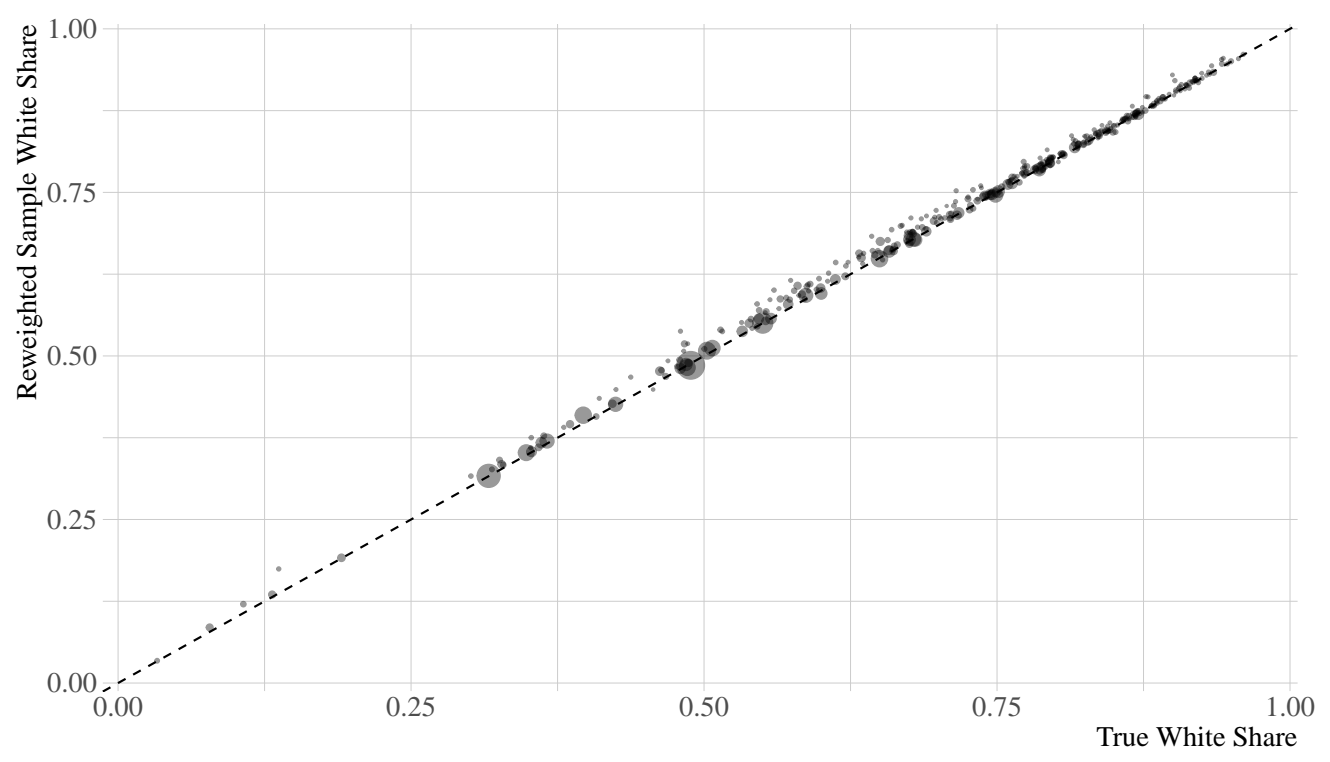

SI Figure S7: Reweighted Sample MSA White Share vs. True MSA White Share

Notes:We plot the MSA white share constructed from reweighting our sample of devices against the true share of the population that is white in each MSA. Each point represents an MSA. The size of each point is proportional to the MSA's population. Note that the sample share white in an MSA is constructed by taking the weighted sum across each observed device's home geohash7 share white. The weights are defined as in equation 4 and are explicitly constructed to recover MSA summary statistics. 


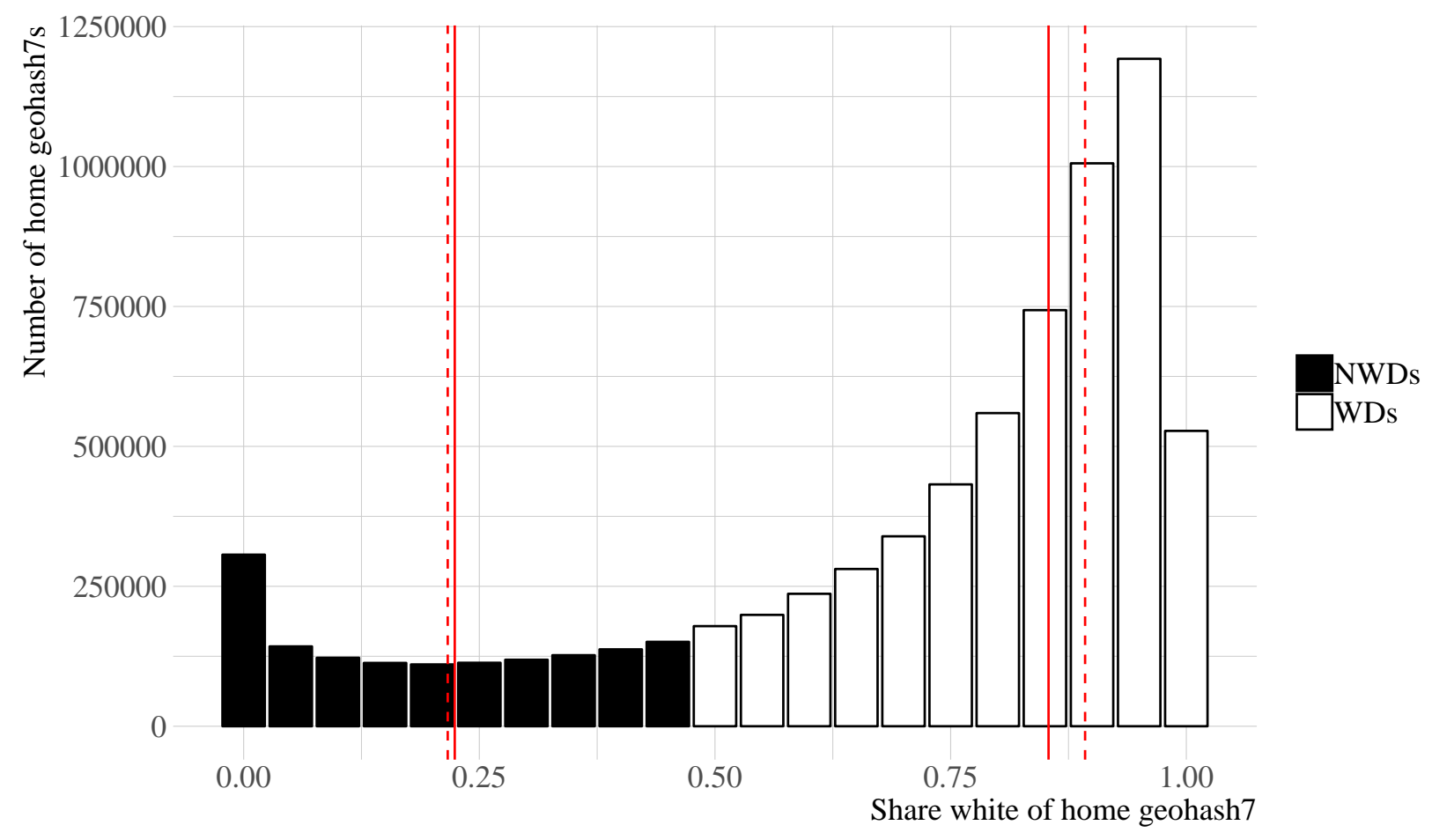

SI Figure S8: Home Geohash7 Percent White Histogram by Majority Race

Notes: Figure plots the number of WD and NWD home geohash7s by the share white of the home geohash7. The mean and median by majority race of the home geohash7 are represented by solid and dashed red lines respectively. The mean and median share white of NWD home geohash7s are both 0.22 and of WD home geohash7s are 0.85 and 0.89 respectively. 


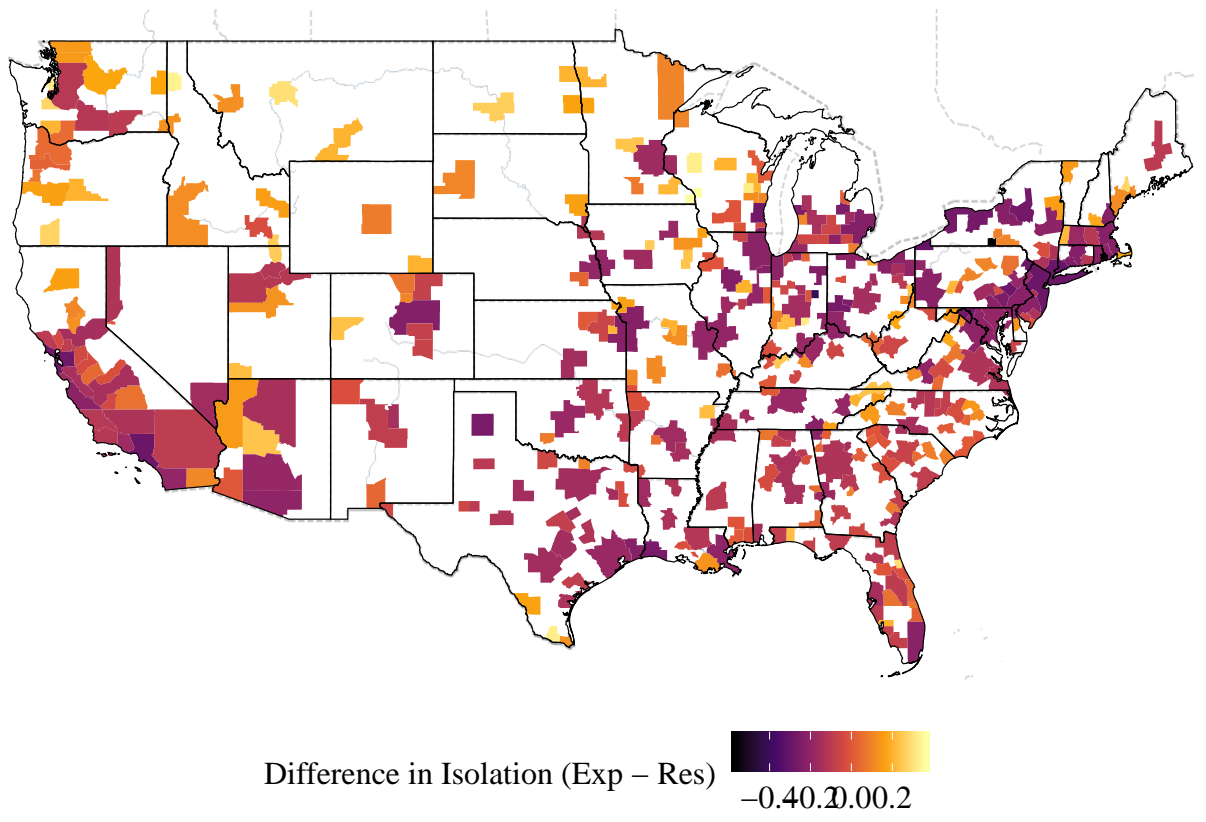

SI Figure S9: Difference Between Experienced and Residential isolation by MSA Notes: We color each MSA relative to the difference of experienced minus residential isolation. 


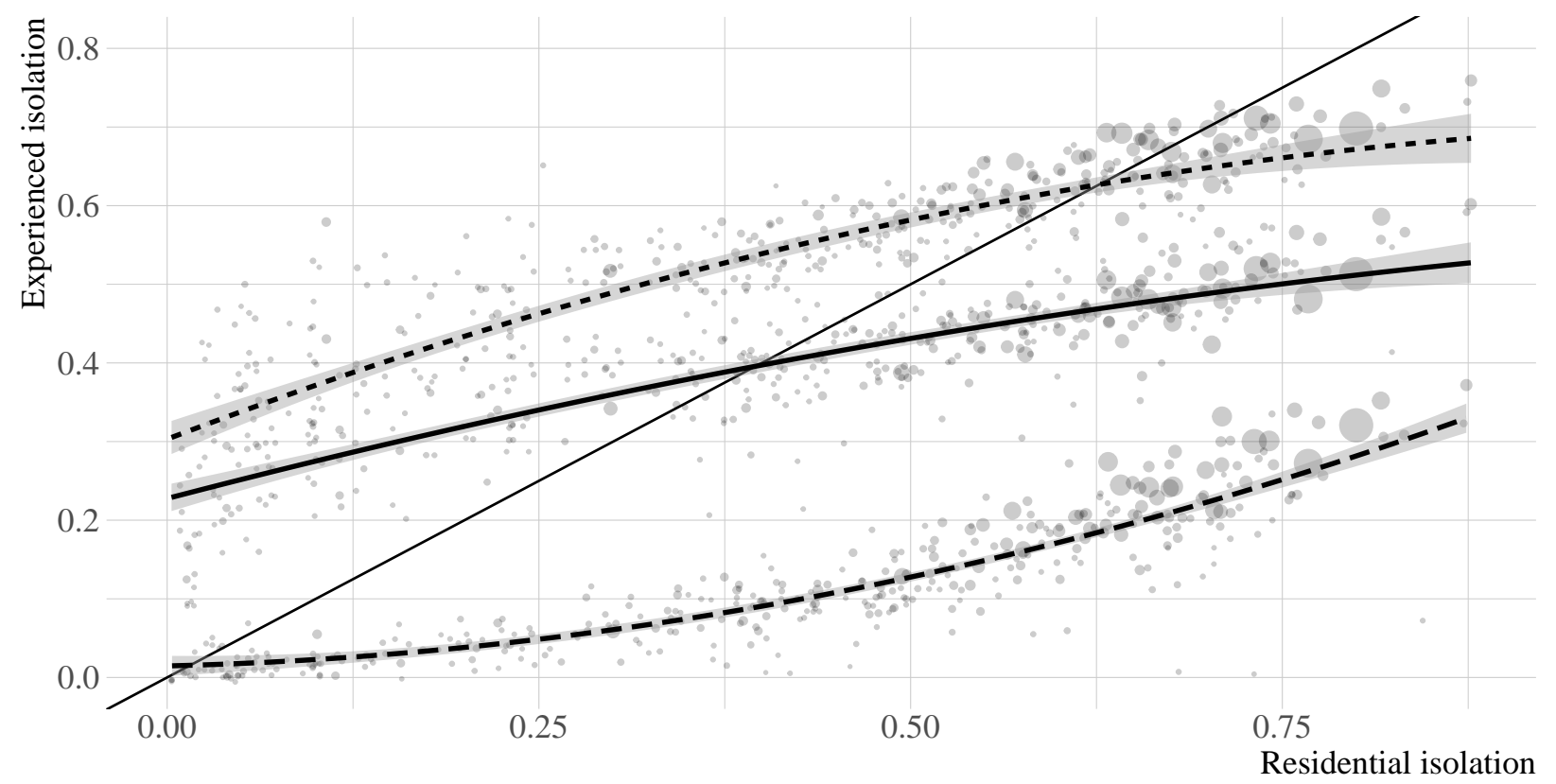

- Baseline - - Within home tract - - Outside home tract

SI Figure S10: Experienced vs Residential Isolation Relative to Devices' Homes

Notes: We plot three specifications of experienced isolation against residential isolation with each point representing an MSA. The within and outside home tract specifications only include exposures in geohash7s within or outside individuals' home census tract. The size of each point is proportional to the MSA's population. We plot the 45 degree line and fit local polynomials to the data. 


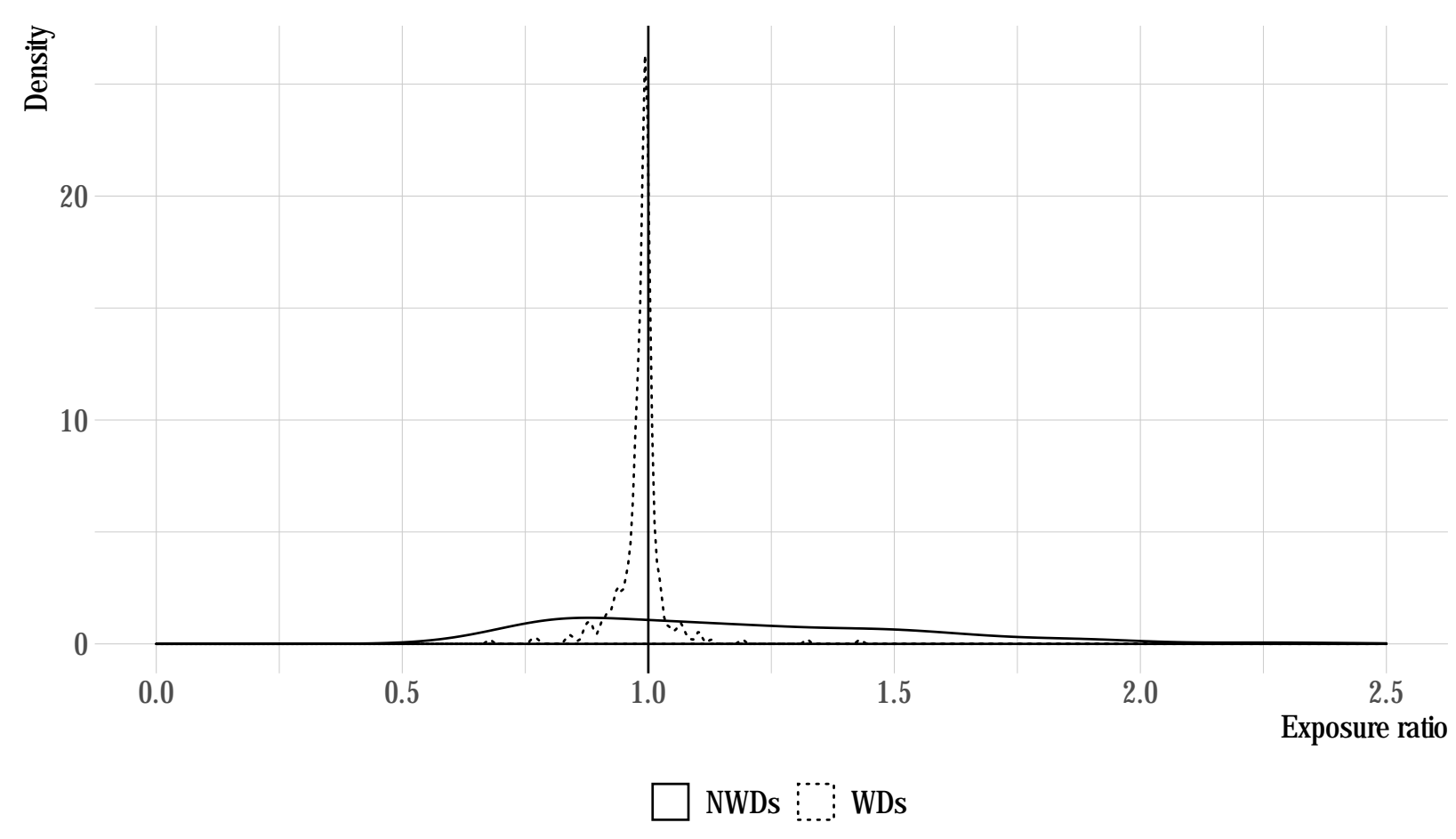

SI Figure S11: Experienced / Residential Exposure for WDs and NWDs

Notes: We plot the distribution of exposure ratios for all WDs and NWDs in our sample. The exposure ratio is $\hat{S}_{i} / \hat{r}_{c(i)}$, the exposure to WDs under the experienced measure over the exposure to WDs for the residential measure. There is much more variation in exposures for NWDs than WDs, suggesting that the primary mechanism for the greater integration we measure relative to residential isolation is driven by NWD exposures. 


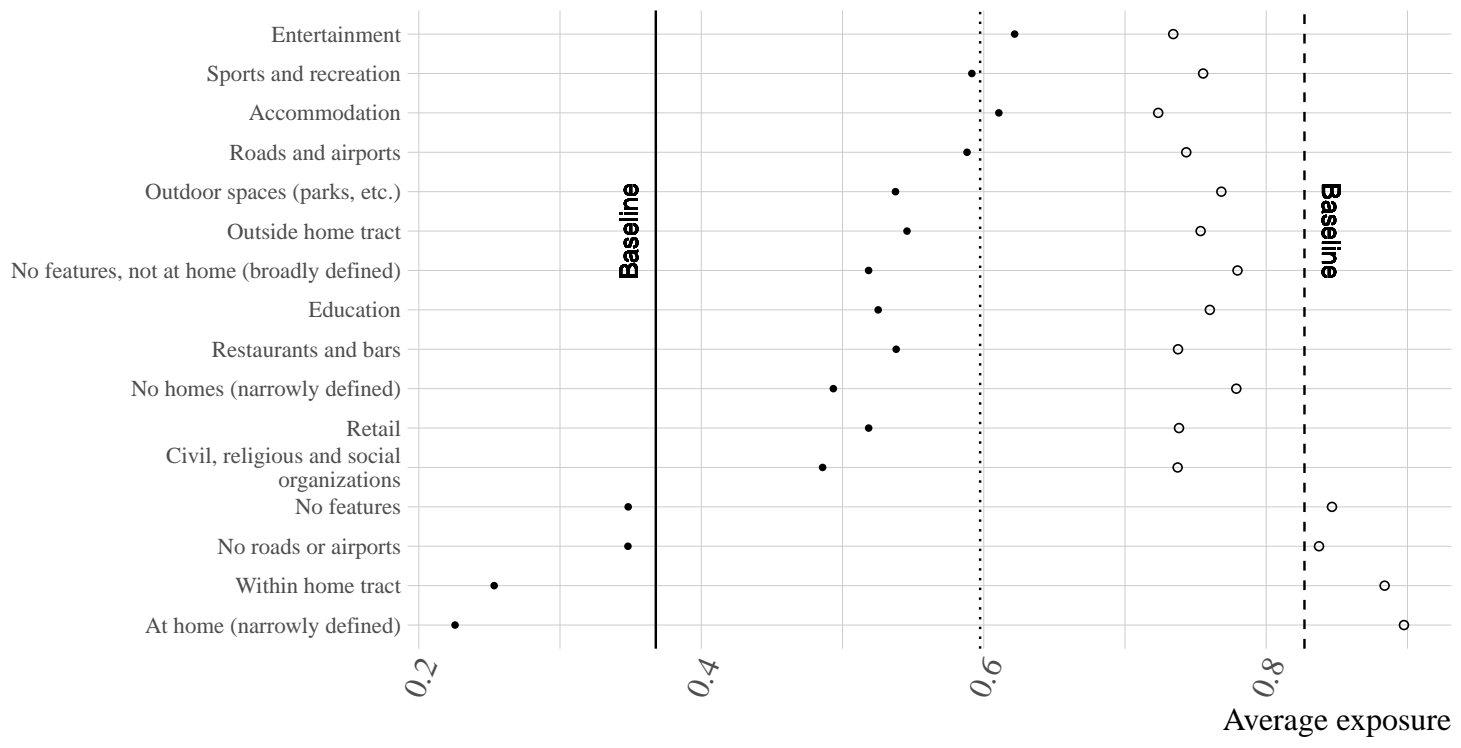

- NWD ○WD

\section{SI Figure S12: Exposure to WDs in Different Features, Decomposition by Race}

Notes: The vertical lines show mean exposures in our baseline specification. The population weighted mean across MSAs of exposure for WDs and NWDs is represented by open and filled points respectively. The distance between any pair of points represents the isolation index in that feature. If the points overlap, isolation is zero. If the WD and NWD populations were contributing equally to their change in exposure, the points would meet at the dotted line splitting the difference between the baseline estimates. 

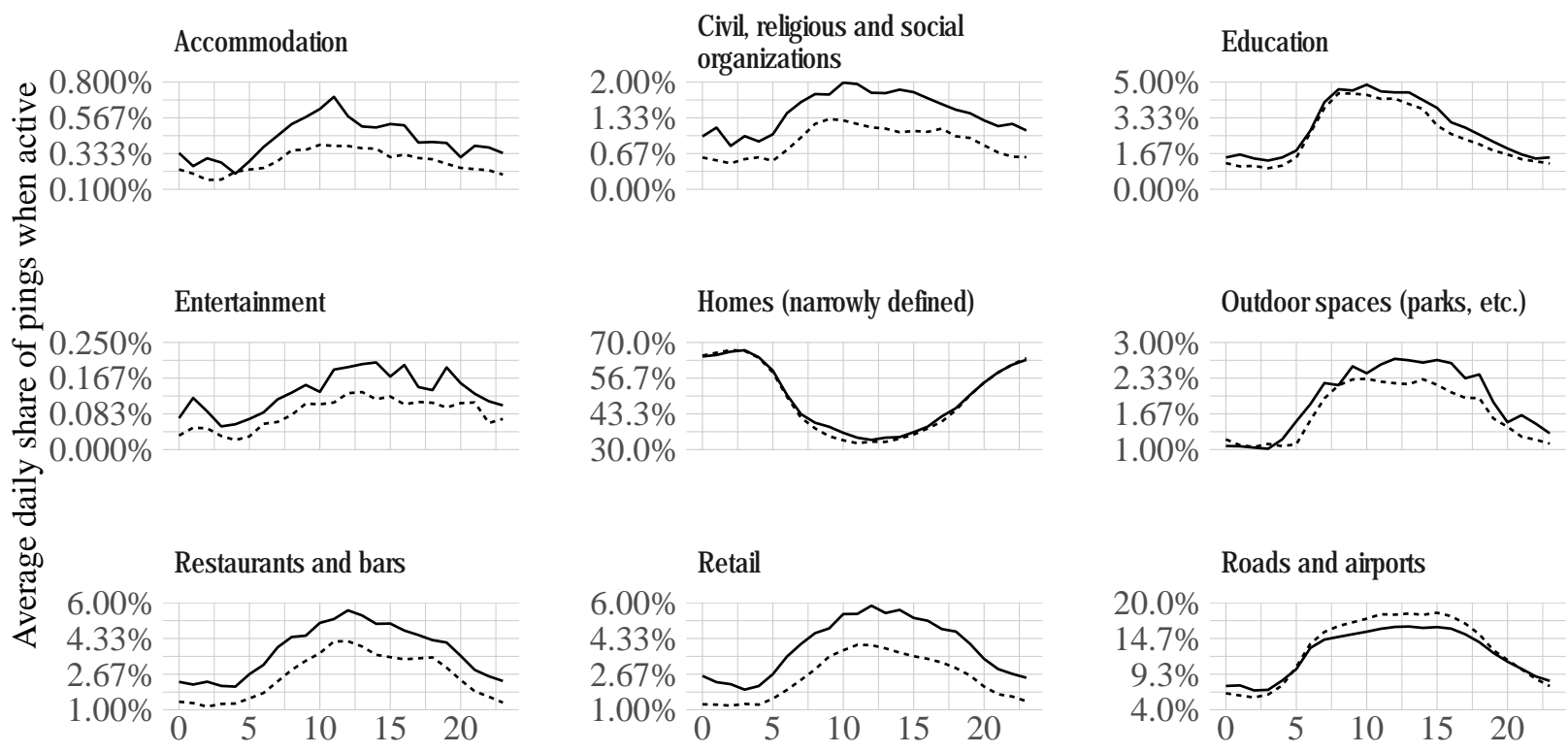

- NWD $\cdots$ WD

\section{SI Figure S13: Average Share of Pings in Features by Device Group}

Notes: We plot the average share of pings in each feature for WDs and NWDs by hour. The solid and dashes lines depict the average for NWDs and WDs respectively. For several features there are interesting differences between groups: NWDs spend more time at civil, religious and social organizations, restaurants and bars, retail establishments and outdoor spaces but less time on roads and at airports. We note that time spent in one's home geohash7, however, is similar for both groups. 


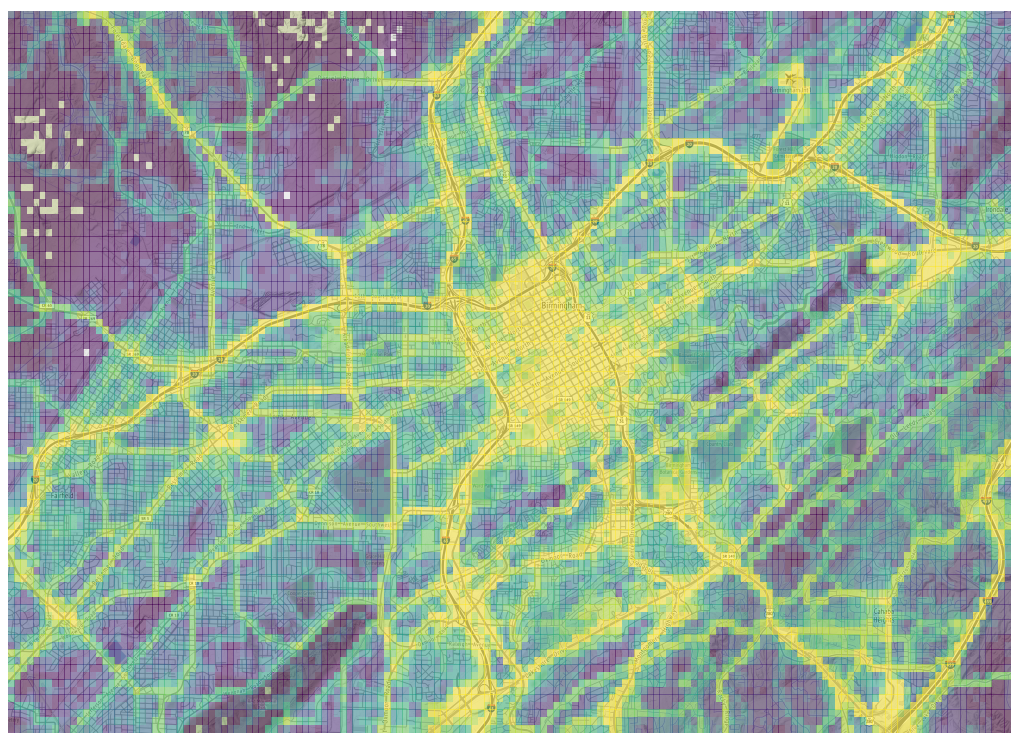

SI Figure S14: Activity in Birmingham, AL

Notes: We depict the level of activity in pings across geohash7s in Birmingham, AL. The number of pings increases as the color moves from blue to yellow. Activity seems to be concentrated on roads and in the central area of the city.

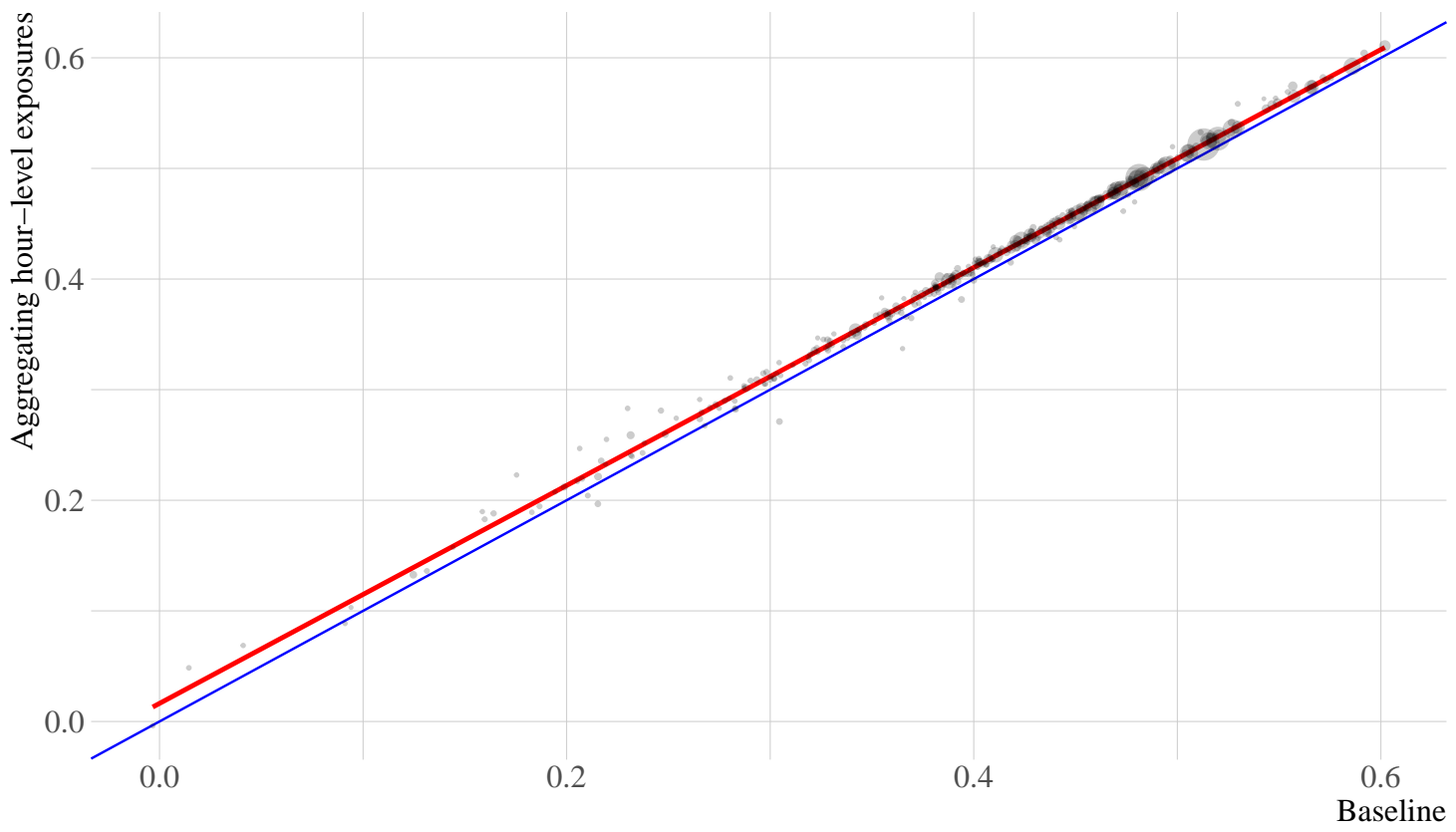

SI Figure S15: Isolation with Hour-level Exposures vs. Baseline

Notes: We plot the specification of experienced isolation using hour-level exposures defined for each geohash7-hour against baseline. Each point represents an MSA. The size of each point is proportional to the MSA's population. We plot the 45 degree line and a linear fit to the data. 
SI Table S1: InfoUSA NAICS6 Categories and Combined Feature Category

\begin{tabular}{|c|c|c|c|}
\hline Combined category & NAICS6 Category & \# of items & Share of all items in infoUSA dataset \\
\hline Retail & Supermarkets/Other Grocery (Exc Convenience) Strs & 90850 & $0.58 \%$ \\
\hline Retail & Pharmacies \& Drug Stores & 73215 & $0.47 \%$ \\
\hline Retail & Convenience Stores & 72947 & $0.47 \%$ \\
\hline Retail & Used Merchandise Stores & 62472 & $0.4 \%$ \\
\hline Retail & All Other General Merchandise Stores & 59747 & $0.38 \%$ \\
\hline Retail & Gift, Novelty \& Souvenir Stores & 54629 & $0.35 \%$ \\
\hline Retail & Women's Clothing Stores & 40032 & $0.26 \%$ \\
\hline Retail & Beer, Wine \& Liquor Stores & 39694 & $0.25 \%$ \\
\hline Retail & Other Clothing Stores & 33709 & $0.22 \%$ \\
\hline Retail & Retail Bakeries & 29162 & $0.19 \%$ \\
\hline Retail & Hobby, Toy \& Game Stores & 26008 & $0.17 \%$ \\
\hline Retail & Department Stores (Except Discount Dept Stores) & 24993 & $0.16 \%$ \\
\hline Retail & Optical Goods Stores & 22419 & $0.14 \%$ \\
\hline Retail & Hardware Stores & 22188 & $0.14 \%$ \\
\hline Retail & All Other Specialty Food Stores & 21094 & $0.13 \%$ \\
\hline Retail & Food (Health) Supplement Stores & 20193 & $0.13 \%$ \\
\hline Retail & All Other Health \& Personal Care Stores & 17774 & $0.11 \%$ \\
\hline Retail & Book Stores & 15910 & $0.1 \%$ \\
\hline Retail & Clothing Accessories Stores & 15578 & $0.1 \%$ \\
\hline Retail & Office Supplies \& Stationery Stores & 12213 & $0.08 \%$ \\
\hline Retail & Paint \& Wallpaper Stores & 10619 & $0.07 \%$ \\
\hline Retail & Children's \& Infants' Clothing Stores & 9963 & $0.06 \%$ \\
\hline Retail & Electronic Shopping & 9871 & $0.06 \%$ \\
\hline Retail & Men's Clothing Stores & 9420 & $0.06 \%$ \\
\hline Retail & Meat Markets & 9398 & $0.06 \%$ \\
\hline Restaurants_bars & Full-Service Restaurants & 607719 & $3.89 \%$ \\
\hline Restaurants_bars & Snack \& Nonalcoholic Beverage Bars & 66575 & $0.43 \%$ \\
\hline Restaurants_bars & Limited-Service Restaurants & 16778 & $0.11 \%$ \\
\hline Civil_social_religious_organizations & Religious Organizations & 386741 & $2.47 \%$ \\
\hline Civil_social_religious_organizations & Civil \& Social Organizations & 64645 & $0.41 \%$ \\
\hline Education & Elementary \& Secondary Schools & 174380 & $1.12 \%$ \\
\hline Education & Colleges, Universities \& Professional Schools & 27442 & $0.18 \%$ \\
\hline Education & Libraries \& Archives & 26059 & $0.17 \%$ \\
\hline Education & Museums & 19108 & $0.12 \%$ \\
\hline Accommodation & Hotels (Except Casino Hotels) \& Motels & 70789 & $0.45 \%$ \\
\hline Accommodation & All Other Traveler Accommodation & 12420 & $0.08 \%$ \\
\hline Accommodation & Bed-\&-Breakfast Inns & 10338 & $0.07 \%$ \\
\hline Sports_recreation & Fitness \& Recreational Sports Centers & 65877 & $0.42 \%$ \\
\hline Entertainment & Motion Picture Theaters (Except Drive-Ins) & 8763 & $0.06 \%$ \\
\hline Entertainment & Theater Companies \& Dinner Theaters & 6484 & $0.04 \%$ \\
\hline
\end{tabular}


SI Table S2: Home Census Tract Summary Statistics for Devices in the Sample.

\begin{tabular}{lrr}
\hline & US mean & Sample mean \\
\hline Female & 0.508 & 0.509 \\
Bachelor's Degree & 0.114 & 0.121 \\
Median Age & 37.385 & 37.431 \\
Median Income (in 1000s of USD) & 28.618 & 29.727 \\
Population in Poverty & 0.133 & 0.124 \\
Unemployment Rate & 0.039 & 0.038 \\
\hline
\end{tabular}

Notes: We aggregate the demographics for the home census tracts in which we observe devices. Data are collected from the 2010 census. Columns show US averages as well as mean for the unweighted device sample. Including sample weights as described in Section 2.4 allows us to exactly recover US averages with the device sample. 
SI Table S3: Summary Statistics for Measures of Activity of Devices in Sample.

\begin{tabular}{lrr}
\hline & Median & Mean \\
\hline Number of days active & 51.00 & 56.92 \\
Number of hours / active day & 7.10 & 9.45 \\
Number of geohash7s visited / active day & 9.68 & 22.95 \\
Number of pings / active day & 33.88 & 86.84 \\
Percent of pings at home (narrowly defined) & 36.79 & 42.15 \\
Number of geohash7s visited overall & 195.00 & 720.85 \\
\hline
\end{tabular}

Notes: All statistics are weighted using the sample weights described in Section 2.4. An active day is a day on which we see at least one ping for the device. 
SI Table S4: Summary of Variables and Sources

\begin{tabular}{|c|c|c|}
\hline Variable & Description & Source \\
\hline Median Age & Median Age & 2010 ACS variable B01002_001 \\
\hline Median Income & Median Income In The Past 12 Months (In 2010 Inflation-Adjusted Dollars) & 2010 ACS variable B06011_001 \\
\hline Population in Poverty & $\begin{array}{l}\text { Count Of Individuals With Income Below Poverty Level For The Past } 12 \\
\text { Months }\end{array}$ & 2010 ACS variable B17001_002 \\
\hline Unemployment Count & Unemployment Count & $\begin{array}{l}\text { Sum of } 2010 \text { ACS variables } \\
\text { B17005_006, } \\
\text { B17005_017 and B17005_022 }\end{array}$ \\
\hline Black Alone & Single Race Non-Hispanic Black Population Count & $\begin{array}{l}2010 \text { Decennial Census variable } \\
\text { P009006 }\end{array}$ \\
\hline Black Alone or in Combination & Single Or Multiracial Non-Hispanic Black Population Count & $\begin{array}{lrr}\text { Sum of } 2010 & \text { Decennial Census } \\
\text { variables } & \text { P009013, } & \text { P009018 } \\
\text { P009019, } & \text { P009020, } & \text { P009021 } \\
\text { P009029, } & \text { P009030, } & \text { P009031, } \\
\text { P009032, } & \text { P009039, } & \text { P009040 } \\
\text { P009041, } & \text { P009042, } & \text { P009043 } \\
\text { P009044, } & \text { P009050, } & \text { P009051 } \\
\text { P009052, } & \text { P009053, } & \text { P009054 } \\
\text { P009055, } & \text { P009060, } & \text { P009061 } \\
\text { P009062, } & \text { P009063, } & \text { P009066 } \\
\text { P009067, } & \text { P009068, } & \text { P009069 } \\
\text { P009071 and P009073 } & \end{array}$ \\
\hline Total Population & Total Population & $\begin{array}{l}2010 \text { Decennial Census variable } \\
\text { P009001 }\end{array}$ \\
\hline White Alone & Single Race Non-Hispanic White Population Count & $\begin{array}{l}2010 \text { Decennial Census variable } \\
\text { P009005 }\end{array}$ \\
\hline Population Density & Population per square mile & $\begin{array}{l}2010 \text { Decennial Census variables } \\
\text { P009001 and SUBHD0303 }\end{array}$ \\
\hline Public Transit Use & Share of working population using public transprotation to get to work & 2010 ACS variable B08101 \\
\hline Share with Bachelors's & Share of population with at least a Bachelor's degree & $\begin{array}{l}2010 \text { ACS variables B06009_005 } \\
\text { and B06009_006 }\end{array}$ \\
\hline Black Income Mobility & $\begin{array}{l}\text { share of black individuals born in the } 25 \text { th percentile of the income distribution } \\
\text { who make it to the top quintile }\end{array}$ & $\begin{array}{l}\text { Average Chetty et al.'s (2018) } \\
\text { pooled by race county estimate } \\
\text { kfr_top20_black_pooled_p25 }\end{array}$ \\
\hline White Income Mobility & $\begin{array}{l}\text { share of white individuals born in the } 25 \text { th percentile of the income distribution } \\
\text { who make it to the top quintile }\end{array}$ & $\begin{array}{l}\text { Average Chetty et al.'s (2018) } \\
\text { pooled by race county estimate } \\
\text { kfr_top20_white_pooled_p25 }\end{array}$ \\
\hline
\end{tabular}


SI Table S5: Experienced and Residential Isolation by MSA

\begin{tabular}{|c|c|c|c|c|c|}
\hline MSA & Exp & Res & MSA & Exp & Res \\
\hline Abilene, TX & 0.30 & 0.46 & Lansing, MI & 0.42 & 0.48 \\
\hline Akron, $\mathrm{OH}$ & 0.46 & 0.61 & Laredo, TX & 0.22 & 0.05 \\
\hline Albany, GA & 0.47 & 0.54 & Las Cruces, NM & 0.39 & 0.35 \\
\hline Albany, NY & 0.48 & 0.68 & Las Vegas, NV & 0.46 & 0.62 \\
\hline Albuquerque, NM & 0.43 & 0.51 & Lawrence, KS & 0.31 & 0.10 \\
\hline Alexandria, LA & 0.44 & 0.58 & Lawton, OK & 0.32 & 0.24 \\
\hline Allentown, PA & 0.48 & 0.68 & Lebanon, PA & 0.41 & 0.44 \\
\hline Altoona, PA & -0.00 & 0.00 & Lewiston, ID & 0.54 & 0.41 \\
\hline Amarillo, TX & 0.48 & 0.73 & Lewiston, ME & 0.50 & 0.23 \\
\hline Ames, IA & 0.35 & 0.10 & Lexington, KY & 0.36 & 0.41 \\
\hline Anchorage, AK & 0.40 & 0.44 & Lima, OH & 0.44 & 0.46 \\
\hline Anderson, IN & 0.41 & 0.41 & Lincoln, NE & 0.30 & 0.27 \\
\hline Anderson, SC & 0.37 & 0.36 & Little Rock, AR & 0.49 & 0.65 \\
\hline Ann Arbor, MI & 0.43 & 0.47 & Logan, UT & 0.34 & 0.08 \\
\hline Anniston, AL & 0.43 & 0.45 & Longview, TX & 0.38 & 0.41 \\
\hline Appleton, WI & 0.42 & 0.21 & Longview, WA & 0.24 & 0.03 \\
\hline Asheville, NC & 0.36 & 0.21 & Los Angeles, CA & 0.48 & 0.77 \\
\hline Athens, GA & 0.41 & 0.39 & Louisville, KY & 0.45 & 0.63 \\
\hline Atlanta, GA & 0.51 & 0.63 & Lubbock, TX & 0.39 & 0.49 \\
\hline Atlantic City, NJ & 0.45 & 0.58 & Lynchburg, VA & 0.39 & 0.43 \\
\hline Auburn, AL & 0.36 & 0.32 & Macon, GA & 0.47 & 0.58 \\
\hline Augusta, GA & 0.44 & 0.46 & Madera, CA & 0.59 & 0.61 \\
\hline Austin, TX & 0.44 & 0.60 & Madison, WI & 0.40 & 0.40 \\
\hline Bakersfield, CA & 0.55 & 0.68 & Manchester, NH & 0.36 & 0.18 \\
\hline Baltimore, MD & 0.52 & 0.71 & Manhattan, KS & 0.42 & 0.42 \\
\hline Bangor, ME & 0.53 & 0.63 & Mankato, MN & 0.09 & 0.02 \\
\hline Barnstable Town, MA & 0.30 & 0.13 & Mansfield, $\mathrm{OH}$ & 0.21 & 0.36 \\
\hline Baton Rouge, LA & 0.47 & 0.58 & McAllen, TX & 0.43 & 0.11 \\
\hline Battle Creek, MI & 0.43 & 0.48 & Medford, OR & 0.33 & 0.06 \\
\hline Bay City, MI & 0.04 & 0.03 & Memphis, TN & 0.52 & 0.66 \\
\hline Beaumont, TX & 0.50 & 0.72 & Merced, CA & 0.38 & 0.34 \\
\hline Bellingham, WA & 0.38 & 0.23 & Miami, FL & 0.49 & 0.71 \\
\hline Bend, OR & 0.21 & 0.01 & Michigan City, IN & 0.43 & 0.51 \\
\hline Billings, MT & 0.40 & 0.20 & Midland, TX & 0.38 & 0.48 \\
\hline Binghamton, NY & 0.22 & 0.12 & Milwaukee, WI & 0.60 & 0.88 \\
\hline Birmingham, AL & 0.57 & 0.71 & Minneapolis, MN & 0.41 & 0.58 \\
\hline Bismarck, ND & 0.28 & 0.03 & Missoula, MT & 0.18 & 0.05 \\
\hline Blacksburg, VA & 0.13 & 0.02 & Mobile, AL & 0.49 & 0.64 \\
\hline Bloomington, IL & 0.34 & 0.13 & Modesto, CA & 0.44 & 0.47 \\
\hline Bloomington, IN & 0.32 & 0.10 & Monroe, LA & 0.51 & 0.63 \\
\hline Boise City, ID & 0.34 & 0.22 & Monroe, MI & 0.32 & 0.23 \\
\hline Boston, MA & 0.45 & 0.68 & Montgomery, AL & 0.48 & 0.62 \\
\hline Boulder, CO & 0.39 & 0.29 & Morgantown, WV & 0.01 & 0.05 \\
\hline Bowling Green, KY & 0.32 & 0.37 & Morristown, TN & 0.27 & 0.07 \\
\hline Bremerton, WA & 0.37 & 0.05 & Mount Vernon, WA & 0.36 & 0.21 \\
\hline Bridgeport, CT & 0.47 & 0.76 & Muncie, IN & 0.41 & 0.82 \\
\hline Brownsville, TX & 0.42 & 0.29 & Muskegon, MI & 0.51 & 0.75 \\
\hline Brunswick, GA & 0.40 & 0.53 & Myrtle Beach, SC & 0.33 & 0.24 \\
\hline Buffalo, NY & 0.52 & 0.78 & Napa, CA & 0.35 & 0.39 \\
\hline Burlington, NC & 0.45 & 0.53 & Naples, FL & 0.48 & 0.55 \\
\hline Burlington, VT & 0.16 & 0.01 & Nashville, TN & 0.45 & 0.63 \\
\hline Canton, OH & 0.38 & 0.48 & New Haven, CT & 0.48 & 0.72 \\
\hline Cape Coral, FL & 0.45 & 0.52 & New Orleans, LA & 0.45 & 0.65 \\
\hline Cape Girardeau, MO & 0.48 & 0.42 & New York, NY & 0.51 & 0.80 \\
\hline Carson City, NV & 0.45 & 0.44 & Niles, MI & 0.55 & 0.73 \\
\hline Casper, WY & 0.09 & 0.01 & North Port, FL & 0.42 & 0.52 \\
\hline Cedar Rapids, IA & 0.27 & 0.13 & Norwich, CT & 0.38 & 0.49 \\
\hline Champaign, IL & 0.33 & 0.52 & Ocala, FL & 0.39 & 0.39 \\
\hline Charleston, SC & 0.36 & 0.44 & Ocean City, NJ & 0.33 & 0.34 \\
\hline Charleston, WV & 0.33 & 0.38 & Odessa, TX & 0.39 & 0.44 \\
\hline Charlotte, NC & 0.47 & 0.62 & Ogden, UT & 0.38 & 0.50 \\
\hline
\end{tabular}

Notes: We report baseline estimates of experienced and residential isolation for each Metropolitan Statistical Area in alphabetical order here and in Tables $\mathbf{S 6}$ and $\mathbf{S 7}$. 
SI Table S6: Experienced and Residential Isolation by MSA: Continued

\begin{tabular}{|c|c|c|c|c|c|}
\hline MSA & Exp & Res & MSA & Exp & Res \\
\hline Charlottesville, VA & 0.30 & 0.23 & Oklahoma City, OK & 0.44 & 0.62 \\
\hline Chattanooga, TN & 0.46 & 0.67 & Olympia, WA & 0.40 & 0.13 \\
\hline Cheyenne, WY & 0.30 & 0.10 & Omaha, NE & 0.49 & 0.68 \\
\hline Chicago, IL & 0.52 & 0.73 & Orlando, FL & 0.46 & 0.55 \\
\hline Chico, CA & 0.39 & 0.28 & Oshkosh, WI & 0.37 & 0.05 \\
\hline Cincinnati, $\mathrm{OH}$ & 0.47 & 0.67 & Owensboro, KY & 0.34 & 0.10 \\
\hline Clarksville, TN & 0.39 & 0.38 & Oxnard, CA & 0.55 & 0.72 \\
\hline Cleveland, $\mathrm{OH}$ & 0.56 & 0.78 & Palm Bay, FL & 0.36 & 0.33 \\
\hline Cleveland, TN & 0.22 & 0.07 & Palm Coast, FL & 0.31 & 0.02 \\
\hline Coeur d'Alene, ID & 0.58 & 0.25 & Panama City, FL & 0.35 & 0.42 \\
\hline College Station, TX & 0.41 & 0.53 & Parkersburg, WV & 0.19 & 0.01 \\
\hline Colorado Springs, CO & 0.45 & 0.53 & Pascagoula, MS & 0.49 & 0.51 \\
\hline Columbia, MO & 0.30 & 0.10 & Pensacola, FL & 0.39 & 0.48 \\
\hline Columbia, SC & 0.45 & 0.52 & Peoria, IL & 0.46 & 0.69 \\
\hline Columbus, GA & 0.48 & 0.65 & Philadelphia, PA & 0.53 & 0.74 \\
\hline Columbus, IN & 0.37 & 0.05 & Phoenix, AZ & 0.52 & 0.70 \\
\hline Columbus, $\mathrm{OH}$ & 0.50 & 0.66 & Pine Bluff, AR & 0.55 & 0.70 \\
\hline Corpus Christi, TX & 0.42 & 0.55 & Pittsburgh, PA & 0.43 & 0.64 \\
\hline Corvallis, OR & 0.22 & 0.15 & Pittsfield, MA & 0.28 & 0.07 \\
\hline Crestview, FL & 0.32 & 0.10 & Pocatello, ID & 0.37 & 0.39 \\
\hline Cumberland, MD & 0.25 & 0.09 & Port St. Lucie, FL & 0.41 & 0.39 \\
\hline Dallas, TX & 0.48 & 0.64 & Portland, ME & 0.23 & 0.12 \\
\hline Dalton, GA & 0.40 & 0.39 & Portland, OR & 0.34 & 0.30 \\
\hline Danville, IL & 0.41 & 0.40 & Poughkeepsie, NY & 0.44 & 0.58 \\
\hline Danville, VA & 0.45 & 0.41 & Prescott, AZ & 0.30 & 0.06 \\
\hline Davenport, IA & 0.36 & 0.36 & Providence, RI & 0.49 & 0.70 \\
\hline Dayton, $\mathrm{OH}$ & 0.56 & 0.82 & Provo, UT & 0.29 & 0.16 \\
\hline Decatur, AL & 0.40 & 0.48 & Pueblo, CO & 0.43 & 0.49 \\
\hline Decatur, IL & 0.44 & 0.52 & Punta Gorda, FL & 0.27 & 0.07 \\
\hline Deltona, FL & 0.38 & 0.40 & Racine, WI & 0.47 & 0.50 \\
\hline Denver, CO & 0.48 & 0.71 & Raleigh, NC & 0.41 & 0.44 \\
\hline Des Moines, IA & 0.37 & 0.54 & Rapid City, SD & 0.28 & 0.18 \\
\hline Detroit, MI & 0.59 & 0.82 & Reading, PA & 0.59 & 0.87 \\
\hline Dothan, AL & 0.38 & 0.36 & Redding, CA & 0.21 & 0.04 \\
\hline Dover, DE & 0.39 & 0.25 & Reno, NV & 0.40 & 0.53 \\
\hline Dubuque, IA & 0.19 & 0.03 & Richmond, VA & 0.46 & 0.58 \\
\hline Duluth, MN & 0.39 & 0.30 & Riverside, CA & 0.48 & 0.57 \\
\hline Durham, NC & 0.43 & 0.51 & Roanoke, VA & 0.46 & 0.66 \\
\hline Eau Claire, WI & 0.36 & 0.03 & Rochester, MN & 0.36 & 0.15 \\
\hline El Centro, CA & 0.48 & 0.37 & Rochester, NY & 0.57 & 0.83 \\
\hline El Paso, TX & 0.34 & 0.39 & Rockford, IL & 0.46 & 0.53 \\
\hline Elizabethtown, KY & 0.38 & 0.27 & Rocky Mount, NC & 0.43 & 0.29 \\
\hline Elkhart, IN & 0.46 & 0.43 & Rome, GA & 0.39 & 0.35 \\
\hline Elmira, NY & 0.14 & 0.70 & Sacramento, CA & 0.53 & 0.68 \\
\hline Erie, PA & 0.35 & 0.65 & Saginaw, MI & 0.52 & 0.76 \\
\hline Eugene, OR & 0.20 & 0.04 & Salem, OR & 0.44 & 0.41 \\
\hline Evansville, IN & 0.39 & 0.41 & Salinas, CA & 0.53 & 0.74 \\
\hline Fairbanks, AK & 0.29 & 0.23 & Salisbury, MD & 0.37 & 0.44 \\
\hline Fargo, ND & 0.27 & 0.10 & Salt Lake City, UT & 0.43 & 0.54 \\
\hline Farmington, NM & 0.44 & 0.46 & San Angelo, TX & 0.37 & 0.49 \\
\hline Fayetteville, AR & 0.44 & 0.45 & San Antonio, TX & 0.49 & 0.65 \\
\hline Fayetteville, NC & 0.40 & 0.40 & San Diego, CA & 0.47 & 0.67 \\
\hline Flagstaff, AZ & 0.47 & 0.62 & San Francisco, CA & 0.42 & 0.70 \\
\hline Flint, MI & 0.57 & 0.74 & San Jose, CA & 0.42 & 0.57 \\
\hline Florence, AL & 0.30 & 0.23 & San Luis Obispo, CA & 0.28 & 0.39 \\
\hline Florence, SC & 0.38 & 0.30 & Sandusky, OH & 0.38 & 0.18 \\
\hline Fond du Lac, WI & 0.16 & 0.04 & Santa Barbara, CA & 0.43 & 0.54 \\
\hline Fort Collins, CO & 0.36 & 0.29 & Santa Cruz, CA & 0.57 & 0.74 \\
\hline Fort Smith, AR & 0.40 & 0.50 & Santa Fe, NM & 0.44 & 0.59 \\
\hline Fort Wayne, IN & 0.53 & 0.76 & Santa Rosa, CA & 0.37 & 0.47 \\
\hline Fresno, CA & 0.47 & 0.62 & Savannah, GA & 0.43 & 0.51 \\
\hline
\end{tabular}

Notes: We report baseline estimates of experienced and residential isolation for each Metropolitan Statistical Area in alphabetical order here and in Tables $\mathbf{S} 5$ and $\mathbf{S 7}$ 
SI Table S7: Experienced and Residential Isolation by MSA: Continued

\begin{tabular}{|c|c|c|c|c|c|}
\hline MSA & Exp & Res & MSA & Exp & Res \\
\hline Gadsden, AL & 0.44 & 0.54 & Scranton, PA & 0.40 & 0.35 \\
\hline Gainesville, FL & 0.39 & 0.51 & Seattle, WA & 0.39 & 0.49 \\
\hline Gainesville, GA & 0.47 & 0.62 & Sebastian, FL & 0.40 & 0.33 \\
\hline Glens Falls, NY & 0.18 & 0.02 & Sheboygan, WI & 0.21 & 0.10 \\
\hline Goldsboro, NC & 0.41 & 0.39 & Sherman, TX & 0.42 & 0.33 \\
\hline Grand Forks, ND & 0.27 & 0.06 & Shreveport, LA & 0.46 & 0.63 \\
\hline Grand Junction, CO & 0.33 & 0.10 & Sioux City, IA & 0.45 & 0.56 \\
\hline Grand Rapids, MI & 0.44 & 0.58 & Sioux Falls, SD & 0.28 & 0.12 \\
\hline Great Falls, MT & 0.32 & 0.03 & South Bend, IN & 0.47 & 0.65 \\
\hline Greeley, CO & 0.45 & 0.52 & Spartanburg, SC & 0.40 & 0.47 \\
\hline Green Bay, WI & 0.39 & 0.38 & Spokane, WA & 0.22 & 0.04 \\
\hline Greensboro, NC & 0.47 & 0.58 & Springfield, IL & 0.41 & 0.58 \\
\hline Greenville, NC & 0.42 & 0.30 & Springfield, MA & 0.54 & 0.72 \\
\hline Greenville, SC & 0.38 & 0.38 & Springfield, MO & 0.12 & 0.01 \\
\hline Gulfport, MS & 0.41 & 0.40 & Springfield, $\mathrm{OH}$ & 0.44 & 0.69 \\
\hline Hagerstown, MD & 0.30 & 0.57 & St. Cloud, MN & 0.29 & 0.05 \\
\hline Hanford, CA & 0.44 & 0.48 & St. George, UT & 0.27 & 0.05 \\
\hline Harrisburg, PA & 0.51 & 0.67 & St. Joseph, MO & 0.23 & 0.07 \\
\hline Harrisonburg, VA & 0.42 & 0.24 & St. Louis, MO & 0.57 & 0.76 \\
\hline Hartford, CT & 0.51 & 0.74 & State College, PA & 0.30 & 0.21 \\
\hline Hattiesburg, MS & 0.40 & 0.40 & Steubenville, $\mathrm{OH}$ & 0.42 & 0.20 \\
\hline Hickory, NC & 0.32 & 0.23 & Stockton, CA & 0.45 & 0.49 \\
\hline Hinesville, GA & 0.44 & 0.36 & Sumter, SC & 0.41 & 0.36 \\
\hline Holland, MI & 0.43 & 0.32 & Syracuse, NY & 0.53 & 0.75 \\
\hline Honolulu, HI & 0.38 & 0.66 & Tallahassee, FL & 0.39 & 0.48 \\
\hline Hot Springs, AR & 0.29 & 0.24 & Tampa, FL & 0.46 & 0.61 \\
\hline Houma, LA & 0.36 & 0.23 & Terre Haute, IN & 0.16 & 0.06 \\
\hline Houston, TX & 0.48 & 0.66 & Texarkana, TX & 0.37 & 0.41 \\
\hline Huntington, WV & 0.25 & 0.22 & Toledo, $\mathrm{OH}$ & 0.47 & 0.67 \\
\hline Huntsville, AL & 0.47 & 0.59 & Topeka, KS & 0.44 & 0.49 \\
\hline Idaho Falls, ID & 0.23 & 0.07 & Trenton, NJ & 0.49 & 0.63 \\
\hline Indianapolis, IN & 0.50 & 0.65 & Tucson, AZ & 0.49 & 0.70 \\
\hline Iowa City, IA & 0.38 & 0.15 & Tulsa, OK & 0.45 & 0.56 \\
\hline Ithaca, NY & 0.32 & 0.23 & Tuscaloosa, AL & 0.46 & 0.49 \\
\hline Jackson, MI & 0.38 & 0.60 & Tyler, TX & 0.43 & 0.58 \\
\hline Jackson, MS & 0.51 & 0.61 & Utica, NY & 0.40 & 0.67 \\
\hline Jackson, TN & 0.43 & 0.58 & Valdosta, GA & 0.42 & 0.47 \\
\hline Jacksonville, FL & 0.46 & 0.54 & Vallejo, CA & 0.46 & 0.55 \\
\hline Jacksonville, NC & 0.34 & 0.35 & Victoria, TX & 0.35 & 0.43 \\
\hline Janesville, WI & 0.43 & 0.43 & Vineland, NJ & 0.46 & 0.53 \\
\hline Jefferson City, MO & 0.32 & 0.20 & Virginia Beach, VA & 0.42 & 0.55 \\
\hline Johnson City, TN & 0.25 & 0.10 & Visalia, CA & 0.40 & 0.34 \\
\hline Johnstown, PA & 0.38 & 0.17 & Waco, TX & 0.45 & 0.60 \\
\hline Jonesboro, AR & 0.39 & 0.17 & Warner Robins, GA & 0.40 & 0.31 \\
\hline Joplin, MO & 0.28 & 0.17 & Washington, DC & 0.47 & 0.68 \\
\hline Kalamazoo, MI & 0.44 & 0.49 & Waterloo, IA & 0.43 & 0.61 \\
\hline Kankakee, IL & 0.46 & 0.68 & Wausau, WI & 0.23 & 0.03 \\
\hline Kansas City, MO & 0.51 & 0.73 & Wenatchee, WA & 0.34 & 0.16 \\
\hline Kennewick, WA & 0.47 & 0.57 & Wheeling, WV & 0.24 & 0.13 \\
\hline Killeen, TX & 0.50 & 0.58 & Wichita Falls, TX & 0.38 & 0.43 \\
\hline Kingsport, TN & 0.29 & 0.06 & Wichita, KS & 0.42 & 0.57 \\
\hline Kingston, NY & 0.33 & 0.35 & Williamsport, PA & 0.30 & 0.20 \\
\hline Knoxville, TN & 0.42 & 0.61 & Wilmington, NC & 0.35 & 0.34 \\
\hline Kokomo, IN & 0.27 & 0.42 & Winchester, VA & 0.40 & 0.27 \\
\hline La Crosse, WI & 0.41 & 0.06 & Winston, NC & 0.48 & 0.60 \\
\hline Lafayette, IN & 0.33 & 0.37 & Worcester, MA & 0.39 & 0.50 \\
\hline Lafayette, LA & 0.39 & 0.41 & Yakima, WA & 0.52 & 0.63 \\
\hline Lake Charles, LA & 0.46 & 0.71 & York, PA & 0.50 & 0.71 \\
\hline Lake Havasu City, AZ & 0.37 & 0.22 & Youngstown, $\mathrm{OH}$ & 0.48 & 0.68 \\
\hline Lakeland, FL & 0.43 & 0.37 & Yuba City, CA & 0.40 & 0.28 \\
\hline Lancaster, PA & 0.47 & 0.71 & Yuma, AZ & 0.49 & 0.48 \\
\hline
\end{tabular}

Notes: We report baseline estimates of experienced and residential isolation for each Metropolitan Statistical Area in alphabetical order here and in Tables $\mathbf{S} 5$ and $\mathbf{S 6}$. 
SI Table S8: Regression Coefficients across Samples

\begin{tabular}{lrrrr}
\hline & Baseline & Top 50 & Top 100 & Top 200 \\
\hline Share with Bachelor's & $-0.2491(0.0428)$ & $-0.426(0.1221)$ & $-0.3269(0.0812)$ & $-0.1403(0.0647)$ \\
Median income (thousands) & $-0.0017(0.0005)$ & $-0.0032(0.0014)$ & $-0.0025(0.0008)$ & $-0.0007(0.0008)$ \\
Unemployment rate & $1.7255(0.2842)$ & $1.0394(0.918)$ & $1.8567(0.4544)$ & $1.5621(0.4083)$ \\
White mobility measure & $-0.2302(0.0449)$ & $-0.4903(0.1105)$ & $-0.2561(0.0938)$ & $-0.0848(0.087)$ \\
Black mobility measure & $-0.146(0.105)$ & $-1.3877(0.3774)$ & $-0.5263(0.2158)$ & $0.0567(0.1525)$ \\
log(Population density) & $-0.0015(0.0007)$ & $-0.0044(0.0026)$ & $-0.0003(0.0011)$ & $-0.0013(0.0009)$ \\
Public transit use & $-0.012(0.0026)$ & $-0.0273(0.0073)$ & $-0.0122(0.0052)$ & $0.0015(0.0041)$ \\
Median age & $-0.1236(0.0295)$ & $-0.3949(0.0881)$ & $-0.3376(0.0888)$ & $-0.245(0.104)$ \\
\hline
\end{tabular}

Notes: We report the coefficient and standard error from our baseline population weighted regression of experienced isolation on fifteen residential isolation bin fixed effects and the specified covariate. We also consider the same regression unweighted and estimated on subsamples of the top 50, 100, and 200 most populous MSAs. 
SI Table S9: Experienced Isolation by Feature Type

\begin{tabular}{|c|c|c|c|c|c|c|}
\hline & q5 & Mean & Median & q95 & $\begin{array}{l}\text { Correl. } \\
\text { with } \\
\text { base- } \\
\text { line }\end{array}$ & $\mathrm{N}$ \\
\hline Baseline & 0.323 & 0.459 & 0.477 & 0.557 & 1.000 & 366 \\
\hline \multicolumn{7}{|l|}{ Features } \\
\hline Accommodation & 0.007 & 0.113 & 0.115 & 0.205 & 0.674 & 366 \\
\hline Civil, Religious And Social Organizations & 0.044 & 0.251 & 0.269 & 0.400 & 0.888 & 366 \\
\hline Education & 0.037 & 0.235 & 0.253 & 0.352 & 0.859 & 366 \\
\hline Entertainment & 0.000 & 0.112 & 0.117 & 0.199 & 0.635 & 362 \\
\hline Outdoor Spaces (Parks, Etc.) & 0.050 & 0.231 & 0.259 & 0.356 & 0.858 & 366 \\
\hline Restaurants And Bars & 0.021 & 0.200 & 0.210 & 0.334 & 0.831 & 366 \\
\hline Retail & 0.025 & 0.220 & 0.233 & 0.356 & 0.863 & 366 \\
\hline Roads And Airports & 0.023 & 0.155 & 0.157 & 0.264 & 0.813 & 366 \\
\hline Sports And Recreation & 0.008 & 0.164 & 0.172 & 0.267 & 0.761 & 365 \\
\hline No Features & 0.342 & 0.498 & 0.526 & 0.593 & 0.944 & 366 \\
\hline \multicolumn{7}{|l|}{ Homes } \\
\hline No Features, Not At Home (Broadly Defined) & 0.065 & 0.261 & 0.278 & 0.392 & 0.887 & 366 \\
\hline At Home (Narrowly Defined) & 0.509 & 0.672 & 0.703 & 0.744 & 0.939 & 366 \\
\hline No Homes (Narrowly Defined) & 0.117 & 0.285 & 0.297 & 0.393 & 0.947 & 366 \\
\hline Outside Home Tract & 0.029 & 0.208 & 0.216 & 0.327 & 0.886 & 366 \\
\hline Within Home Tract & 0.450 & 0.630 & 0.662 & 0.713 & 0.961 & 366 \\
\hline
\end{tabular}

Notes: We report summary statistics for different specifications of our measure weighted by MSA population. We consider measure experienced isolation restricted to various features and degrees of home proximity. 
SI Table S10: Robustness

\begin{tabular}{|c|c|c|c|c|c|c|c|}
\hline & & \multicolumn{6}{|c|}{ Experienced Isolation } \\
\hline & & q5 & Mean & Median & q95 & $\begin{array}{l}\text { Correl. } \\
\text { with } \\
\text { base- } \\
\text { line }\end{array}$ & $\begin{array}{l}\text { Correl. } \\
\text { with } \\
\text { Res. } \\
\text { Isola- } \\
\text { tion }\end{array}$ \\
\hline 1 & Baseline & 0.323 & 0.459 & 0.477 & 0.557 & 1.000 & 0.864 \\
\hline \multicolumn{8}{|c|}{ Robustness checks } \\
\hline 2 & No Roads Or Airports & 0.339 & 0.489 & 0.509 & 0.586 & 0.991 & 0.860 \\
\hline 3 & Only Pings $<12 \mathrm{mph}$ & 0.363 & 0.507 & 0.524 & 0.599 & 0.996 & 0.860 \\
\hline 4 & All (L2 Imputation) & 0.244 & 0.390 & 0.409 & 0.481 & 0.908 & 0.843 \\
\hline 5 & All (Infutor imputation) & 0.251 & 0.421 & 0.437 & 0.530 & 0.910 & 0.853 \\
\hline 6 & All (Day-Hour Weighting) & 0.260 & 0.409 & 0.427 & 0.512 & 0.984 & 0.884 \\
\hline 7 & All (Hour-level Exposures) & 0.335 & 0.469 & 0.487 & 0.570 & 0.999 & 0.865 \\
\hline 8 & Without Out-Of-Towners & 0.341 & 0.476 & 0.491 & 0.577 & 0.990 & 0.843 \\
\hline 9 & $\begin{array}{l}\text { Without Top 5\% Active Users In Terms Of } \\
\text { Pings Per Day }\end{array}$ & 0.333 & 0.476 & 0.495 & 0.572 & 0.992 & 0.883 \\
\hline 10 & Exclude Night Hours & 0.305 & 0.441 & 0.459 & 0.542 & 0.999 & 0.863 \\
\hline 11 & All (Without Leave-One-Out Exposures) & 0.384 & 0.499 & 0.506 & 0.596 & 0.937 & 0.754 \\
\hline
\end{tabular}

Notes: We report summary statistics for different specifications of our measure. We consider excluding transportation features like roads, airports, or devices moving fast enough to be considered in transit. We also consider different subsamples of users, weighting schemes, and demographic data sources. 
SI Table S11: Sample Statistics Restricting Exposure During Transportation

\begin{tabular}{lrrr}
\hline & Devices & Geohash7s & Pings \\
\hline Baseline & $17,397,580$ & $98,853,493$ & $101,989,194,959$ \\
No roads or airports & $17,328,912$ & $91,277,728$ & $76,324,902,186$ \\
Only pings $<12 \mathrm{mph}$ & $17,381,896$ & $79,837,642$ & $68,019,968,506$ \\
Only pings $<8 \mathrm{mph}$ & $17,381,803$ & $75,775,799$ & $65,123,085,955$ \\
Only pings $<4 \mathrm{mph}$ & $17,381,553$ & $69,193,133$ & $60,991,437,300$ \\
Only pings $<4 \mathrm{mph} \&$ no roads or airports & $17,307,535$ & $62,639,284$ & $53,991,029,734$ \\
\hline
\end{tabular}

Notes: We remove pings emitted at speeds exceeding different thresholds or on transport infrastructure and report counts of devices, geohash7s, and pings on these subsamples. 
SI Table S12: Summary Statistics for Alternative Measures of Isolation

\begin{tabular}{llllll}
\hline & & Exp. mean & Res. mean & $\begin{array}{l}\text { Correl. with } \\
\text { res. }\end{array}$ & $\begin{array}{l}\text { Correl. with } \\
\text { baseline }\end{array}$ \\
\hline 1 & Baseline & 0.459 & 0.605 & 0.864 & 1.000 \\
Home geohash7 race & & & & \\
\hline 2 & White-nonwhite (63/37) & & & & \\
3 & White-Black (50/50) & 0.456 & 0.598 & 0.860 & 0.988 \\
$4 \quad$ White-Black (70/70) & 0.470 & 0.655 & 0.867 & 0.703 \\
$5 \quad$ White-Black (90/90) & 0.474 & 0.658 & 0.857 & 0.612 \\
& & 0.387 & 0.450 & 0.875 & 0.436 \\
Direct race imputation & & & & \\
\hline $6 \quad$ White-nonwhite (direct) & & & & \\
7 & White-Black (direct) & 0.185 & 0.311 & 0.930 & 0.838 \\
\hline
\end{tabular}

Notes: We report mean experienced and residential isolation along with their correlation under the alternative measures. We also report the correlation of the alternative measures with baseline. All estimates are weighted by MSA population. The specification White/Black (XX/YY) indicates that isolation is estimated between devices from white and black home geohash7s that are considered white if the share white is above XX and considered black if the share black is above YY. Direct means that individuals are assigned the indicated device groups probabalistically. 